\title{
A Generalized Reduced complexity Inertial Navigation System for Unmanned Aerial Vehicles
}

\author{
Emil Fresk, George Nikolakopoulos and Thomas Gustafsson
}

\begin{abstract}
In this article a generic approach to attitude and position estimation, suited for any type of Unmanned Aerial Vehicle will be presented. This will be achieved by establishing a generic framework, which can be extended using adaptive methods to determine the thrust properties of the engines and the mass of the aircraft, while keeping the overall computational complexity of the system low. Furthermore, the effect of magnetic disturbances will be reduced in a novel way by confining the magnetic errors to only affect heading, without compromising the pitch and roll estimation of the system with error based estimation. The efficacy of the proposed framework will be evaluated through extended simulations and experimental validations on a multirotor. Finally, guidelines will be provided towards: a) an implementation with a reduced computational complexity, and b) the utilization of the square-root formulations of the Extended Kalman Filter for extending the dynamical range of the filter.
\end{abstract}

Index Terms-Error estimation, Attitude estimation, Position estimation, Adaptive filters, Inertial navigation system, Global positioning system, Unmanned Aerial Vehicle.

\section{INTRODUCTION}

Unmanned Aerial Vehicles (UAVs), such as the multirotors, have been in focus of research and development, mainly due to their efficiency in completing complex missions and providing a good fundamental base for research. This includes application areas such as forest fire inspection [1], infrastructure inspection, search and rescue missions [2], manipulation of objects and cooperative missions, including cooperative manipulation [3]. In all these applications, accurate attitude and position estimation is essential to complete the mission, since the controllers are based on the information provided by the estimation.

To achieve the desired control performance in an UAV, three main problems arise that need to be solved. The attitude problem [4] and the translation problem, as it has been proven, can be interconnected in a cascade form [5], [6] to achieve the desired performance, whilst keeping the complexity low. The final problem is the fact that the control system should be designed for on board utilization, where attitude and position estimation is required to stabilize the UAV and since these UAVs are quite often very small, the weight of the control system can have a profound impact on the performance of the UAV. Thus, it is desirable to use small embedded systems to

E. Fresk, G. Nikolakopoulos and T. Gustafsson are with the department of Computer Science, Electrical and Space Engineering at Luleå University of Technology, SE-97187, Luleå, Sweden (e-mail: emil.fresk@ltu.se, geonik@1tu.se,tgu@1tu.se).

This work has received funding from the European Unions Horizon 2020 Research and Innovation Programme under the Grant Agreement No.644128, AEROWORKS

(O)2017 IEEE do the estimation and control tasks [7], mainly due to their small size, low weight, low cost and high reliability.

The attitude estimation problem has received extended research focus mainly for estimating the attitude of spacecrafts, where the usage of quaternion error representations [8] has gained significant importance the last years, usually due to its efficiency and smaller number of states. One popular approach is nonlinear filtering, such as the Unscented Kalman Filter (UKF) [9], [10] or the Cubature Kalman Filter (CKF) [11], [12], which was proven to have better convergence, especially when the states have bad initialization. However, this approach indicated equal tracking performance to the Extended Kalman Filter based algorithms [13], [14] after convergence. Moreover, little effort has been made on implementing efficiently from a computational complexity approach, except from utilizing the common algorithms, while there have been only a few approaches in the utilization of the square-root formulation for an extended dynamical range [15], [11], [12].

It should also be noted that significant work has been done, not utilizing error representations nor quaternions, where [16] created two deterministic observers, one termed direct and the second termed passive, that formulated the kinematics directly on $\mathrm{SO}(3)$. These observers were designed based on Lyapunov stability analysis and were shown to be almost globally stable based on the observers' errors through extended proofs, analysis and experiments. Furthermore, [17] developed the proper theory and geometrical framework for designing symmetry-preserving observers on Lie groups, which were applied, as an example, towards inertial navigation. The strong merit of these observers is the fact that they are intrinsically and globally defined whilst, in the particular case of inertial navigation aided by magnetic measurements, are convergent around any trajectory. However, in both of the aforementioned works, it has been assumed that the attitude information could be extracted from the accelerometers, and additionally in the case of [17] that the magnetic measurements are error free. This approach is a sensitive problem, which in [18] has been further examined and analyzed, where in the case of extended dynamical models, this is a possible solution but a more general reference is needed, as it will be discussed in this article. Additionally, in the work to be presented, a framework for solving this problem will be proposed by allowing generalized reference vectors and removing the forced connection with accelerometers.

In the field of position estimation there has not been as much research work as done with the case of attitude estimation, while the standard approach has been to have a two stage estimation schemes, where the attitude is forwarded to a 
position estimator that integrates acceleration and compensates with a Global Positioning System (GPS) to get a position estimate [19]. This approach however, has the drawback of loosing attitude convergence during accelerations, plus that the system does not predict the accelerations. However, the work presented in [20] shows how this combined type of estimation can be utilized on a quadrotor platform together with drag estimation, which highlights the idea but is aimed at a specific platform. We will present a novel approach where the previous two stages and frame dependent approach, has been extended with cross couplings between the attitude and position estimation in order to provide additional information on the physics of the generalized UAV.

The additional parameters needed from the UAV to increase the estimation accuracy, referred to as a parameter adaptive estimation scheme or an Extended State Observer, can be used to minimize the effect of modeling uncertainties, both structured and unstructured as discussed in [21], [22], by improving the model parameters while still being robust to generalized disturbances, such as modeling uncertainties.

The novelty of this article stems from: a) a combined attitude and position estimation scheme designed for embedded systems, b) an adaptive physical model by estimating the generated thrust, independent of the number of engines and mass of the UAV, c) a reduced implementation complexity to minimize computations, and d) a generic estimation algorithm that works as a base for any UAV platform.

The rest of the article is structured as follows. In Section II, an overview of the quaternion algebra and the Generalized Rodrigues Parameters for usage in attitude estimation is presented, while in Section III the overall generic filter formulation is presented. In Section IV the performance of the proposed estimation scheme is being initially evaluated in both extended simulation and experimental verification. In Section $\mathrm{V}$ the results, simplifications and approximations are discussed and finally in Section VI the conclusions are drawn.

$\begin{array}{rll}\text { Abbreviations } & & \\ \hat{\boldsymbol{e}}_{x}, \hat{\boldsymbol{e}}_{y}, \hat{\boldsymbol{e}}_{z}: & \text { Cartesian unit vectors } \\ \boldsymbol{q} & : & \text { Quaternion } \\ \boldsymbol{\delta} \boldsymbol{q} & : & \text { Quaternion error } \\ \boldsymbol{p} & : & \text { Generalized Rodrigues Parameter } \\ \boldsymbol{\delta} \boldsymbol{p} & : & \text { Generalized Rodrigues Parameter error } \\ \boldsymbol{\omega} & : & \text { Rotational rate of the multirotor } \\ \boldsymbol{\delta} \boldsymbol{\omega} & : & \text { Gyroscope bias error } \\ \boldsymbol{x}^{F} & : & \boldsymbol{x} \text { is in the fixed frame of reference } \\ \boldsymbol{x}^{B} & : & \boldsymbol{x} \text { is in the body frame of reference } \\ \mathbf{c}_{x} & : & \text { cos }(x) \\ \mathbf{s}_{x} & : & \text { sin }(x) \\ \theta_{e}^{Y} & : & \text { Error angles around roll, pitch and } \\ & & \text { yaw respectively } \\ \text { lat } & : & \text { Latitude Coordinate } \\ \text { lon } & : & \text { Longitude Coordinate } \\ \text { UAV } & : & \text { Unmanned Aerial Vehicle } \\ \text { VTOL } & : & \text { Vertical Take-Off and Landing } \\ \text { MEMS } & : & \text { Micro Electro Mechanical System } \\ \text { INS } & : & \text { Inertial Navigation System } \\ \text { GPS } & : & \text { Global Positioning System } \\ \text { EKF } & : & \text { Extended Kalman Filter } \\ \text { SR-EKF } & : & \text { Square-root Extended Kalman Filter } \\ \text { UKF } & : & \text { Unscented Kalman Filter }\end{array}$

$\begin{array}{rll}\text { SR-UKF } & : & \text { Square-root Unscented Kalman Filter } \\ \text { CKF } & : & \text { Cubature Kalman Filter } \\ \text { SR-CKF } & : & \text { Square-root Cubature Kalman Filter } \\ \text { RMSE } & : & \text { Root-Mean-Square Error } \\ \text { GRP } & : & \text { Generalized Rodrigues Parameter } \\ \text { MCU } & : & \text { Micro Controller Unit } \\ \text { DSP } & : & \text { Digital Signal Processor } \\ \text { FPU } & : & \text { Floating Point Unit } \\ \text { ADC } & : & \text { Analog to Digital Converter } \\ \text { PWM } & : & \text { Pulse Width Modulation } \\ \text { DMA } & : & \text { Direct Memory Access } \\ \text { SOC } & : & \text { System On a Chip } \\ \text { UART } & : & \text { Universal Asynchronous } \\ & & \text { Receiver/Transmitter } \\ \text { CAN } & : & \text { Controller Area Network } \\ \text { USB } & : & \text { Universal Serial Bus } \\ \text { I C } & : & \text { Inter-Integrated Circuit } \\ \text { VCP } & : & \text { Virtual Com Port } \\ \text { ESD } & : & \text { Electro Static Discharge } \\ \text { RSSI } & : & \text { Received Signal Strength Indication } \\ \text { ESC } & : & \text { Electronic Speed Controllers } \\ \text { ISR } & : & \text { Interrupt Service Routine } \\ \text { RF } & : & \text { Radio Frequency }\end{array}$

\section{QUATERnions \& GENERALIZED RodRIGUES PARAMETERS}

For consistency reasons, and for establishing the necessary supporting mathematical background for the proposed modeling and control scheme, this Section is going to present the basic algebraic concepts behind the idea of quaternions and Generalized Rodrigues Parameters (GRPs). For a more comprehensive analysis and an in depth description of these mathematical tools, the reader is refereed to the following publications [23], [24] for quaternions and [25] for GRPs.

A quaternion is a hyper complex number of rank 4, which can be represented in many ways, while:

$$
\begin{aligned}
\boldsymbol{q} & =q_{0}+q_{1} \boldsymbol{i}+q_{2} \boldsymbol{j}+q_{3} \boldsymbol{k}, \\
\boldsymbol{q} & =\left[\begin{array}{llll}
q_{0} & q_{1} & q_{2} & q_{3}
\end{array}\right]^{T}
\end{aligned}
$$

represent two of the most popular approaches where $\boldsymbol{q} \in \mathbb{H}$, $\boldsymbol{i}, \boldsymbol{j}, \boldsymbol{k} \in \mathbb{C}$ are the complex unit vectors, $\left\{q_{0}, q_{1}, q_{2}, q_{3}\right\} \in \mathbb{R}$. The quaternion units from $q_{1}$ to $q_{3}$ are called the vector part, or also the complex part, of the quaternion, while $q_{0}$ is the scalar part (sometimes denoted $q_{4}$ or $w$ ). The multiplication of two quaternions $\boldsymbol{p}, \boldsymbol{q}$ is defined by the Kronecker product, denoted as $\otimes$ :

$$
\begin{aligned}
\boldsymbol{p} \otimes \boldsymbol{q}= & {\left[\begin{array}{l}
p_{0} q_{0}-p_{1} q_{1}-p_{2} q_{2}-p_{3} q_{3} \\
p_{0} q_{1}+p_{1} q_{0}+p_{2} q_{3}-p_{3} q_{2} \\
p_{0} q_{2}-p_{1} q_{3}+p_{2} q_{0}+p_{3} q_{1} \\
p_{0} q_{3}+p_{1} q_{2}-p_{2} q_{1}+p_{3} q_{0}
\end{array}\right], } \\
\boldsymbol{p} \otimes \boldsymbol{q}= & \left.Q \begin{array}{cccc}
p_{0} & -p_{1} & -p_{2} & -p_{3} \\
p_{1} & p_{0} & -p_{3} & p_{2} \\
p_{2} & p_{3} & p_{0} & -p_{1} \\
p_{3} & -p_{2} & p_{1} & p_{0}
\end{array}\right]\left[\begin{array}{l}
q_{0} \\
q_{1} \\
q_{2} \\
q_{3}
\end{array}\right] \\
= & \bar{Q}(\boldsymbol{q}) \boldsymbol{p}=\left[\begin{array}{cccc}
q_{0} & -q_{1} & -q_{2} & -q_{3} \\
q_{1} & q_{0} & q_{3} & -q_{2} \\
q_{2} & -q_{3} & q_{0} & q_{1} \\
q_{3} & q_{2} & -q_{1} & q_{0}
\end{array}\right]\left[\begin{array}{l}
p_{0} \\
p_{1} \\
p_{2} \\
p_{3}
\end{array}\right] .
\end{aligned}
$$

If $\boldsymbol{p}$ represents one rotation and $\boldsymbol{q}$ represents another rotation $\boldsymbol{p} \otimes \boldsymbol{q}$ represents the combined rotation. It's important to note 
that quaternion multiplication is non-commutative, just as rotations are non-commutative. The norm/length of a quaternion is defined, just as for any complex number, as

$$
\operatorname{Norm}(\boldsymbol{q})=\|\boldsymbol{q}\|=\sqrt{q_{0}^{2}+q_{1}^{2}+q_{2}^{2}+q_{3}^{2}} .
$$

We assume that all quaternions have unitary length and thus are called unit quaternions. The complex conjugate of a quaternion has the same definition as normal complex numbers. The sign of the complex part is switched as

$$
\operatorname{Conj}(\boldsymbol{q})=\boldsymbol{q}^{*}=\left[\begin{array}{llll}
q_{0} & -q_{1} & -q_{2} & -q_{3}
\end{array}\right]^{T} .
$$

The inverse of a quaternion is defined as

$$
\operatorname{Inv}(\boldsymbol{q})=\boldsymbol{q}^{-1}=\frac{\boldsymbol{q}^{*}}{\|\boldsymbol{q}\|^{2}}
$$

just as the normal inverse of a complex number. Moreover, if the length of the quaternion is unitary then the inverse is the same as the conjugate. The derivative of a quaternion requires some algebraic manipulation and can be represented as [24]:

$$
\dot{\boldsymbol{q}}_{\omega}(\boldsymbol{q}, \boldsymbol{\omega})=\frac{1}{2} \boldsymbol{q} \otimes\left[\begin{array}{l}
0 \\
\boldsymbol{\omega}
\end{array}\right]=\frac{1}{2} Q(\boldsymbol{q})\left[\begin{array}{l}
0 \\
\boldsymbol{\omega}
\end{array}\right]
$$

in case that the angular velocity vector is in the fixed frame of reference, and as

$$
\dot{\boldsymbol{q}}_{\omega}(\boldsymbol{q}, \boldsymbol{\omega})=\frac{1}{2}\left[\begin{array}{l}
0 \\
\boldsymbol{\omega}
\end{array}\right] \otimes \boldsymbol{q}=\frac{1}{2} \bar{Q}(\boldsymbol{q})\left[\begin{array}{l}
0 \\
\boldsymbol{\omega}
\end{array}\right]
$$

if the angular velocity vector is in the body frame of reference, where $\boldsymbol{\omega}=\left[\omega_{x}, \omega_{y}, \omega_{z}\right]^{T}$. If a quaternion is a unit quaternion it can be used as a rotation operator. However the transformation is not built up by only one quaternion multiplication but two, the normal and its conjugate, as

$$
\boldsymbol{w}=\boldsymbol{q} \otimes\left[\begin{array}{l}
0 \\
\mathbf{v}
\end{array}\right] \otimes \boldsymbol{q}^{*}
$$

This rotates the vector $\mathbf{v}$ from the fixed frame to the body frame represented by $\mathbf{q}$. This rotation, in equation (8), can be rewritten by replacing $\mathbf{v}$ with the Euclidean basis vectors, as it is being displayed in the following equations:

$$
\begin{aligned}
& R_{x}(\boldsymbol{q})=\boldsymbol{q} \otimes\left[\begin{array}{l}
0 \\
1 \\
0 \\
0
\end{array}\right] \otimes \boldsymbol{q}^{*}=\left[\begin{array}{c}
q_{0}^{2}+q_{1}^{2}-q_{2}^{2}-q_{3}^{2} \\
2\left(q_{1} q_{2}+q_{0} q_{3}\right) \\
2\left(q_{1} q_{3}-q_{0} q_{2}\right)
\end{array}\right], \\
& R_{y}(\boldsymbol{q})=\boldsymbol{q} \otimes\left[\begin{array}{l}
0 \\
0 \\
1 \\
0
\end{array}\right] \otimes \boldsymbol{q}^{*}=\left[\begin{array}{c}
2\left(q_{1} q_{2}-q_{0} q_{3}\right) \\
q_{0}^{2}-q_{1}^{2}+q_{2}^{2}-q_{3}^{2} \\
2\left(q_{2} q_{3}+q_{0} q_{1}\right)
\end{array}\right], \\
& R_{z}(\boldsymbol{q})=\boldsymbol{q} \otimes\left[\begin{array}{l}
0 \\
0 \\
0 \\
1
\end{array}\right] \otimes \boldsymbol{q}^{*}=\left[\begin{array}{c}
2\left(q_{1} q_{3}+q_{0} q_{2}\right) \\
2\left(q_{2} q_{3}-q_{0} q_{1}\right) \\
q_{0}^{2}-q_{1}^{2}-q_{2}^{2}+q_{3}^{2}
\end{array}\right] .
\end{aligned}
$$

It should be noted that in the examined case only the vector part of the quaternion has been extracted, resulting in a rotation matrix, which rotates a point in a fixed coordinate system, as

$$
R(\boldsymbol{q})=\left[\begin{array}{lll}
R_{x}(\boldsymbol{q}) & R_{y}(\boldsymbol{q}) & R_{z}(\boldsymbol{q})
\end{array}\right]
$$

When rotating a coordinate system, compared to when rotating point in a coordinate system, is the same operation as equation (12) with the opposite angle sign which provides:

$$
R(\boldsymbol{q})^{T}=\left[\begin{array}{l}
R_{x}(\boldsymbol{q})^{T} \\
R_{y}(\boldsymbol{q})^{T} \\
R_{z}(\boldsymbol{q})^{T}
\end{array}\right]
$$

while the same result arises when conjugating the quaternion in equation (8). This is also directly connected to the general rotation matrix from Euler angles as

$$
R=\left[\begin{array}{ccc}
\mathrm{c}_{\theta} \mathrm{c}_{\psi} & \mathrm{c}_{\phi} \mathrm{s}_{\psi}+\mathrm{s}_{\phi} \mathrm{s}_{\theta} \mathrm{c}_{\psi} & \mathrm{s}_{\phi} \mathrm{s}_{\psi}-\mathrm{c}_{\phi} \mathrm{s}_{\theta} \mathrm{c}_{\psi} \\
-\mathrm{c}_{\theta} \mathrm{s}_{\psi} & \mathrm{c}_{\phi} \mathrm{c}_{\psi}-\mathrm{s}_{\phi} \mathrm{s}_{\theta} \mathrm{s}_{\psi} & \mathrm{s}_{\phi} \mathrm{c}_{\psi}+\mathrm{c}_{\phi} \mathrm{s}_{\theta} \mathrm{s}_{\psi} \\
\mathrm{s}_{\theta} & -\mathrm{s}_{\phi} \mathrm{c}_{\theta} & \mathrm{c}_{\phi} \mathrm{c}_{\theta}
\end{array}\right] .
$$

The rotation can also be represented using a rotation vector as

$$
\boldsymbol{q}=\cos \left(\frac{\alpha}{2}\right)+\boldsymbol{u} \sin \left(\frac{\alpha}{2}\right),
$$

where $\boldsymbol{u}$ is the rotation axis (unit vector) and $\alpha$ is the angle of rotation. Using this notation can have many benefits when creating an error or specifying a reference as it has a direct physical connection. Finally, for representing quaternion rotations in a more intuitive manner, the conversion from Euler angles to quaternion and from quaternion to Euler angle can be performed by utilizing the following two equations respectively,

$$
\boldsymbol{q}=\left[\begin{array}{l}
\mathrm{c}_{\phi / 2} \mathrm{c}_{\theta / 2} \mathrm{c}_{\psi / 2}+\mathrm{s}_{\phi / 2} \mathrm{~s}_{\theta / 2} \mathrm{~s}_{\psi / 2} \\
\mathrm{~s}_{\phi / 2} \mathrm{c}_{\theta / 2} \mathrm{c}_{\psi / 2}-\mathrm{c}_{\phi / 2} \mathrm{~s}_{\theta / 2} \mathrm{~s}_{\psi / 2} \\
\mathrm{c}_{\phi / 2} \mathrm{~s}_{\theta / 2} \mathrm{c}_{\psi / 2}+\mathrm{s}_{\phi / 2} \mathrm{c}_{\theta / 2} \mathrm{~s}_{\psi / 2} \\
\mathrm{c}_{\phi / 2} \mathrm{c}_{\theta / 2} \mathrm{~s}_{\psi / 2}-\mathrm{s}_{\phi / 2} \mathrm{~s}_{\theta / 2} \mathrm{c}_{\psi / 2}
\end{array}\right],
$$

and the inverse transform as

$$
\left[\begin{array}{l}
\phi \\
\theta \\
\psi
\end{array}\right]=\left[\begin{array}{c}
\operatorname{atan} 2\left(2\left(q_{0} q_{1}+q_{2} q_{3}\right), 1-2\left(q_{1}^{2}+q_{2}^{2}\right)\right) \\
\operatorname{asin}\left(2\left(q_{0} q_{2}-q_{1} q_{3}\right)\right) \\
\operatorname{atan} 2\left(2\left(q_{0} q_{3}+q_{1} q_{2}\right), 1-2\left(q_{2}^{2}+q_{3}^{2}\right)\right)
\end{array}\right] .
$$

This property is very useful when the aim is to represent the orientation in angles, while retaining the overall dynamics of the system in quaternion form.

A Generalized Rodrigues Parameter (GRP) is different from a quaternion, in the sense that it only has three components, whilst a quaternion has four and a GRP, contrary to quaternions, has a singularity, which can be placed almost arbitrarily, as discussed in [14]. This error representation can be calculated from an error quaternion as

$$
\boldsymbol{\delta} \boldsymbol{p}=f \frac{\boldsymbol{\delta} \boldsymbol{q}_{v}}{a+\delta q_{0}},
$$

where $\delta \boldsymbol{q}_{v}$ is the vector part of the quaternion error, $f \in \mathbb{R}^{+}$ is used to set the scaling of the small angle approximation and $a \in \mathbb{R}^{+}$to set where the singularity is placed, with typical values of $f=4$ and $a=1$, equaling four times the Modified Rodrigues Parameter [9], making the small angle approximation equal the angle, without scaling. The inverse transformation, back to an error quaternion [25], is presented below:

$$
\begin{aligned}
\delta q_{0} & =\frac{-a\|\boldsymbol{\delta} \boldsymbol{p}\|^{2}+f \sqrt{f^{2}+\left(1-a^{2}\right)\|\boldsymbol{\delta} \boldsymbol{p}\|^{2}}}{f^{2}+\|\boldsymbol{\delta} \boldsymbol{p}\|^{2}} \\
\boldsymbol{\delta} \boldsymbol{q}_{v} & =\frac{a+\delta q_{0}}{f} \boldsymbol{\delta} \boldsymbol{p}
\end{aligned}
$$


The dynamics of attitude errors have been thoroughly examined [8], [14], where the second order approximation

$$
\boldsymbol{\delta} \dot{p}=\boldsymbol{\delta} p \times\left(\omega+\frac{1}{2} \delta \omega\right)+\delta \omega,
$$

is the most popular to use with an EKF [8], where $\boldsymbol{\delta} \boldsymbol{p} \in \mathbb{R}^{3}$ is the attitude error, $\boldsymbol{\omega} \in \mathbb{R}^{3}$ is the bias compensated angular rate and $\delta \boldsymbol{\omega} \in \mathbb{R}^{3}$ is the current estimate of the bias error. This comes from the fact that the Jacobian, of the state prediction equations, is not gaining any more information when adding more degrees since the states are close - or equal - to zero.

\section{FILTER FORMULATION}

\section{A. Estimation overview}

In Figure 1 a complete overview of the generic estimation algorithm is presented. The inputs are divided into four categories, 1) reference vectors where $\boldsymbol{r}_{m}^{B}, \boldsymbol{b}_{m}^{B} \in \mathbb{R}^{3}$ are a general reference vector and the magnetic field vector respectively, which can be used as attitude references, 2) IMU which provide the relative information where $\boldsymbol{a}_{m}^{B}, \boldsymbol{\omega}_{m}^{B} \in \mathbb{R}^{3}$ are acceleration and angular rate respectively, 3 ) absolute sensors, some which provide global position information, such as GPS, and some that provides absolute values with respect to the body frame, such as sonars, and 4) actuators to be used as feed forward and parameter estimation in the algorithm where $\boldsymbol{u}_{k} \in \mathbb{R}^{n}$ is the input vector.

With this structure, it is assumed that the following sensors are available: An accelerometer, a gyroscope, a magnetometer, a sensor that provides the general reference vector and absolute sensors, such as GPS, cameras, sonars or laser scanners, to compensate for position drift.

The reference vectors are transformed into error quantities where $\boldsymbol{\theta}_{e, k} \in \mathbb{R}^{3}$ are the error angles and the IMU acceleration data is transformed into the fixed frame of reference, $\boldsymbol{a}_{m}^{F} \in \mathbb{R}^{3}$, as will be presented in Section III-C. These, plus the absolute sensors and the actuator signals, are fed to the SR-EKF, which will be presented in Section III-B, in order to estimate the $\delta \boldsymbol{p}_{k \mid k}, \boldsymbol{\delta} \boldsymbol{\omega}_{k \mid k} \in \mathbb{R}^{3}$, which are the attitude and gyroscope's bias errors respectively to correct the attitude and gyroscope bias estimation, which are $\hat{\boldsymbol{q}}_{k \mid k}, \hat{\boldsymbol{\omega}}_{k \mid k} \in \mathbb{R}^{3}$ respectively. Furthermore, the position, velocity and parameters $\left(\hat{\boldsymbol{p}}_{k \mid k}^{F}, \hat{\boldsymbol{v}}_{k \mid k}^{F} \in \mathbb{R}^{3}, \hat{\boldsymbol{\theta}}_{k \mid k} \in \mathbb{R}^{p}\right)$ are directly estimated.

It should be noted that the entire system works in the fixed North (x), West (y) and Up (z) frame (NWU), if it is desired to work in the North (x), East (y) and Down (z) frame (NED) some conversions will have to be made. Moreover, all the notations and the sub-block analysis, depicted in Figure 1 will be presented in the sequel.

\section{B. Square-Root Extended Kalman Filter}

In this section we present the underlying equations of the SR-EKF [26], [27]. This implementation was chosen to counteract the problem of dynamical range in floating-point arithmetic, where the sum of a small and a large number will equal the large number (rounding away the small number), while the square-root formulation has the merit to effectively double the usable dynamical range.
In the following algorithm, $\operatorname{chol}(X)$ denotes a Cholesky decomposition returning the lower triangular matrix $L$, chol $_{\text {downdate }}(L, X)$ denotes a down date of a Cholesky decomposition $(L)$, utilizing the columns of $X$ as down dating vectors and returning the down dated lower triangular matrix, while $\mathbf{Q R}(X)$ denotes a $\mathrm{QR}$ decomposition returning the upper triangular matrix $R$ from the decomposition.

\section{FILTER INITIALIZATION}

1) Calculate the square-root factor of the starting error covariance matrix:

$$
S_{P,-1 \mid-1}=\operatorname{chol}\left(P_{0}\right) .
$$

2) Introduce the starting state estimate:

$$
\hat{\boldsymbol{x}}_{-1 \mid-1}=\boldsymbol{x}_{0}
$$

\section{UPDATE COVARIANCE MATRICES}

1) Calculate the square-root factor of the process noise covariance:

$$
S_{Q, k}=\operatorname{chol}\left(Q_{k}\right) .
$$

2) Calculate the square-root factor of the observation noise covariance:

$$
S_{R, k}=\operatorname{chol}\left(R_{k}\right)
$$

\section{Prediction Estimate}

1) Predict the state:

$$
\hat{\boldsymbol{x}}_{k \mid k-1}=\boldsymbol{f}\left(\hat{\boldsymbol{x}}_{k-1 \mid k-1}, \boldsymbol{u}_{k}\right) .
$$

2) Estimate the square-root factor of the predicted error covariance matrix:

$$
S_{P, k \mid k-1}=\mathbf{Q R}\left(\left[F_{k} S_{P, k-1 \mid k-1}, S_{Q, k}\right]^{T}\right)^{T},
$$

where

$$
F_{k}=\left.\frac{\partial \boldsymbol{f}}{\partial \boldsymbol{x}}\right|_{\hat{\boldsymbol{x}}_{k-1 \mid k-1}, \boldsymbol{u}_{k}} .
$$

MEASUREMENT UPDATE

1) Subtract the predicted measurement from the true measurement:

$$
\boldsymbol{y}_{k}=\boldsymbol{z}_{k}-\boldsymbol{h}\left(\hat{\boldsymbol{x}}_{k \mid k-1}, \boldsymbol{u}_{k}\right) \text {. }
$$

2) Estimate the square-root factor of the innovation covariance matrix:

$$
S_{S, k}=\mathbf{Q R}\left(\left[H_{k} S_{P, k \mid k-1}, S_{R, k}\right]^{T}\right)^{T}
$$

where

$$
H_{k}=\left.\frac{\partial \boldsymbol{h}}{\partial \boldsymbol{x}}\right|_{\hat{\boldsymbol{x}}_{k \mid k-1}, \boldsymbol{u}_{k}} .
$$

3) Calculate the Kalman gain:

$$
K_{k}=\left(S_{P, k \mid k-1} S_{P, k \mid k-1}^{T}\right) H_{k}^{T}\left(S_{S, k} S_{S, k}^{T}\right)^{-1} .
$$

During this step, it should be highlighted that no explicit inverse is needed, since $S_{S, k}$ is a triangular matrix and forward/backward substitutions replace all inverses. This can 


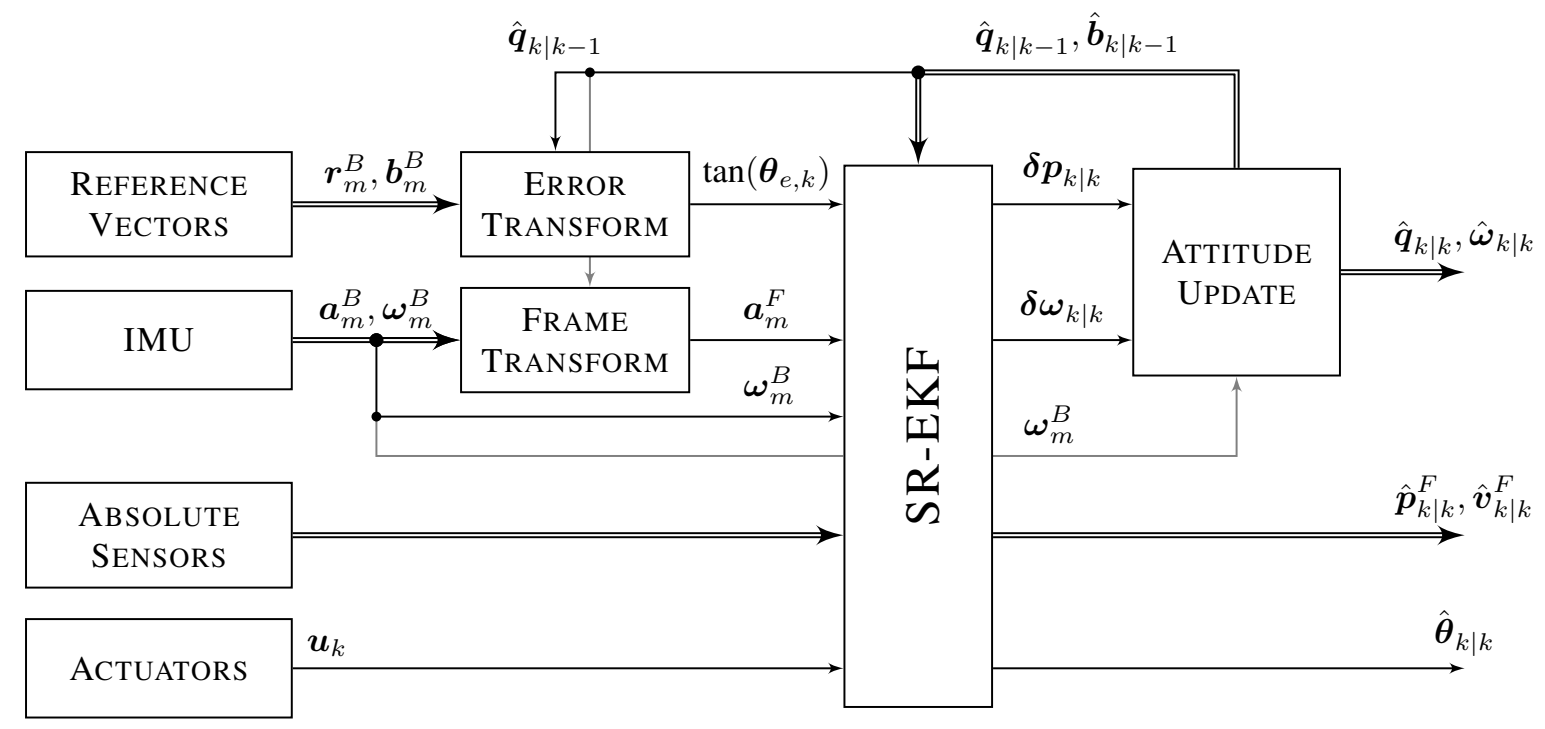

Fig. 1. A complete overview of the entire attitude, position and parameter estimation, from sensors and actuators inputs to estimated state outputs at time instance $k$. The "Reference Vectors"-block represents attitude references consisting of a generic attitude reference and the magnetometer, the "IMU"-block represents the inertial sensors consisting of an accelerometer and gyroscope, the "Absolute Sensors"-block represents sensors such as GPS, Sonars, Barometers etc (application specific). A thin line represents a vector and a double line represents a collection of vectors.

be straight forward solved by solving a triangular system of the form $L X=B$, which is provided by solving $L \boldsymbol{x}=\boldsymbol{b}$, where $\boldsymbol{x}$ is each column in $X$ and $\boldsymbol{b}$ is each column in $B-$ forward or backward substitution depends on if $L$ is lower or upper triangular.

4) Calculate the updated state estimate:

$$
\hat{\boldsymbol{x}}_{k \mid k}=\hat{\boldsymbol{x}}_{k \mid k-1}+K_{k} \boldsymbol{y}_{k} \text {. }
$$

5) Calculate the square-root factor of the corresponding error covariance matrix:

$$
S_{P, k \mid k}=S_{P, k \mid k-1} W_{k},
$$

where

$$
W_{k}=\operatorname{chol}_{\text {downdate }}\left(I,\left(S_{S, k}^{-1} H_{k} S_{P, k \mid k-1}\right)^{T}\right)
$$

and

$$
\operatorname{dim}(I)=\operatorname{dim}\left(S_{P, k \mid k-1}\right) .
$$

If the problem is ill conditioned, another down date algorithm called the QR down date [28], which uses Givens rotations, can be used that has better numerical properties but needs more computations than the Cholesky down date. In this implementation, computational speed has been chosen as the main goal, since the problem of attitude and position estimation is well conditioned.

\section{Attitude model}

The attitude estimation part of the filter is designed to be a Multiplicative EKF, implying that the attitude is propagated as error quantities. This has the advantages of reducing the state vector by one compared to using a full quaternion, since it only represents the error and not the entire state [8], and that the states are guaranteed to be close to zero which will simplify a lot of the system equations in the following analysis.

1) Error representation and states: The attitude error representation chosen is the Generalized Rodrigues Parameter as presented in Section II and from equation (21) also requires the angular rate bias error $\delta \boldsymbol{\omega}$. Using this representation for the dynamics, the following state vector $\hat{\boldsymbol{x}}_{k}^{A}$ arises to represent the attitude error and the bias error:

$$
\hat{\boldsymbol{x}}_{k}^{A}=\left[\begin{array}{c}
\boldsymbol{\delta} \boldsymbol{p}_{k} \\
\boldsymbol{\delta} \boldsymbol{\omega}_{k}
\end{array}\right] .
$$

Assuming that the dynamics are slow during each time step gives the following $0^{\text {th }}$ order integrator, some times called a rectangle rule, to predict the state:

$$
\boldsymbol{f}\left(\hat{\boldsymbol{x}}_{k}, u_{e, k}\right)=\left[\begin{array}{c}
\boldsymbol{\delta} \boldsymbol{p}_{k-1 \mid k-1}+\Delta t \boldsymbol{\delta} \dot{\boldsymbol{p}}_{k-1 \mid k-1} \\
\boldsymbol{\delta} \boldsymbol{\omega}_{k-1 \mid k-1}
\end{array}\right] .
$$

2) Measurement transformation: The main transformation to keep the system computationally simple is to transform the measurements from a general reference vector and the magnetic field vector, directly to error angles.

Assumption 1: Assume there is a vector $\boldsymbol{r}_{m}^{B}$ that can be measured in the body frame of the UAV and which is known in the fixed frame to be used as a reference vector.

By using this reference vector, the error can be calculated using vector algebra to produce the tangent of the error angles as:

$$
\tan (\alpha)=\frac{\hat{\boldsymbol{W}} \cdot \boldsymbol{V}_{r e f \perp}}{\hat{\boldsymbol{W}} \cdot \boldsymbol{V}_{\text {ref }}}
$$

where $\hat{\boldsymbol{W}}$ is the measurement and $\boldsymbol{V}_{\text {ref }}$ is the reference direction as depicted in Figure 3. At small errors this expression is the error states directly in the fixed frame of reference 


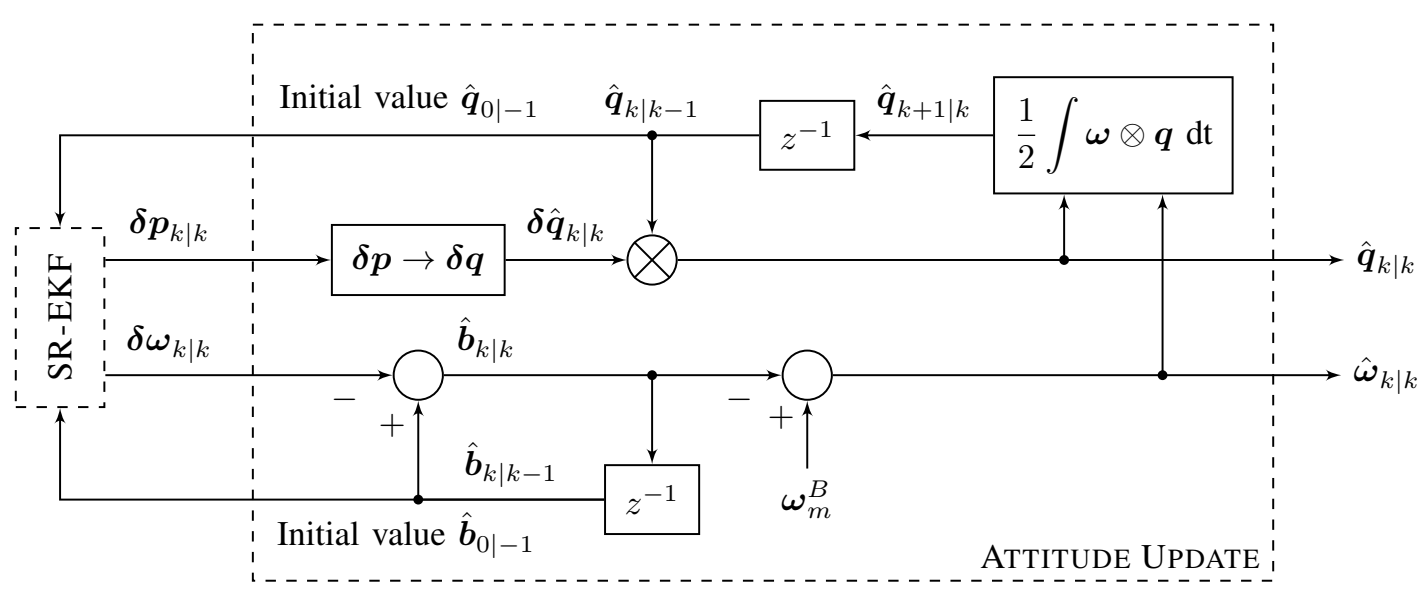

Fig. 2. A complete internal view of the Attitude Update block from Figure 1, including from the estimated error states to attitude.

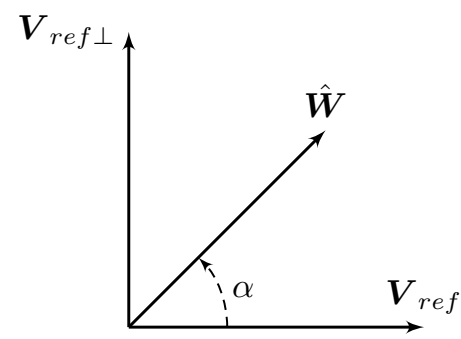

Fig. 3. An illustration of the vector projections in equation (24).

and using this property of vectors the reference and magnetic vector can directly be transformed to error angles as:

$$
\left[\begin{array}{l}
\tan \left(\theta_{e, k}^{R}\right) \\
\tan \left(\theta_{e, k}^{P}\right) \\
\tan \left(\theta_{e, k}^{Y}\right)
\end{array}\right]=\left[\begin{array}{c}
-\frac{\boldsymbol{r}_{m}^{F} \cdot \hat{\boldsymbol{e}}_{y}^{F}}{\boldsymbol{r}_{m}^{F} \cdot \hat{\boldsymbol{e}}_{z}^{F}} \\
\frac{\boldsymbol{r}_{m}^{F} \cdot \hat{\boldsymbol{e}}_{x}^{F}}{\boldsymbol{r}_{m}^{F} \cdot \hat{\boldsymbol{e}}_{z}^{F}} \\
\boldsymbol{b}_{k}^{F} \cdot \hat{\boldsymbol{e}}_{y}^{F} \\
\boldsymbol{b}_{k}^{F} \cdot \hat{\boldsymbol{e}}_{x}^{F}
\end{array}\right],
$$

where $\theta_{e}^{R}, \theta_{e}^{P}$ and $\theta_{e}^{Y} \in \mathbb{R}$ are the error angles around roll, pitch and yaw respectively. Due to practicality, a small change must be made as:

$$
\boldsymbol{\theta}_{e, k}=\left[\begin{array}{c}
\operatorname{atan} 2\left(-\boldsymbol{r}_{m}^{F} \cdot \hat{\boldsymbol{e}}_{y}^{F}, \boldsymbol{r}_{m}^{F} \cdot \hat{\boldsymbol{e}}_{z}^{F}\right) \\
\operatorname{atan} 2\left(\boldsymbol{r}_{m}^{F} \cdot \hat{\boldsymbol{e}}_{x}^{F}, \boldsymbol{r}_{m}^{F} \cdot \hat{\boldsymbol{e}}_{z}^{F}\right) \\
\operatorname{atan} 2\left(\boldsymbol{b}_{k}^{F} \cdot \hat{\boldsymbol{e}}_{y}^{F}, \boldsymbol{b}_{k}^{F} \cdot \hat{\boldsymbol{e}}_{x}^{F}\right)
\end{array}\right],
$$

to counteract the problem of division by zero [29] which could happen in equation (25), which also will be used in the measurement equation presented in the sequel.

Since the magnetic measurement is very susceptible to errors and interference it is desirable to only use this information for correcting the yaw estimation. This guarantees that errors in the magnetic measurement will not translate to errors in roll and pitch, with the added advantage that the local magnetic field can be unknown. This is done by one property of the magnetic field - the horizontal part of the vector always points
North, this is extracted by using the $x$ and $y$ components of the vector to calculate the yaw error. However the measurements are made in the body frame of reference, hence first must be transformed to the fixed frame. This is done using the current attitude estimate, as:

$$
\begin{aligned}
\boldsymbol{r}_{m}^{F} & =R(\hat{\boldsymbol{q}})^{T} R(\boldsymbol{\delta} \boldsymbol{p})^{T} \boldsymbol{r}_{m}^{B}, \\
\boldsymbol{b}_{m}^{F} & =R(\hat{\boldsymbol{q}})^{T} R(\boldsymbol{\delta} \boldsymbol{p})^{T} \boldsymbol{b}_{m}^{B},
\end{aligned}
$$

where $R(\cdots)^{T}$ is the attitude matrix transformation from equation (13). Using these transformations the attitude input vector is reduced from a size of 6 to 3 entries, greatly reducing the computational complexity of the algorithm. Since the measurement now is the attitude error states in the fixed frame of reference the transformation is defined as:

$$
\boldsymbol{h}\left(\hat{\boldsymbol{x}}_{k}\right)=R\left(\hat{\boldsymbol{q}}_{k}\right)^{T} R\left(\boldsymbol{\delta} \boldsymbol{p}_{k}\right)^{T} \boldsymbol{\delta} \boldsymbol{p}_{k} .
$$

The last step of the attitude update procedure is to update the true attitude and gyroscope bias with the error estimates, this effectively moves the current estimation by the mean of the error, as presented in Figure 2. This is known as the "reset" of the filter, where all the states are zeroed [14].

The attitude update procedure follows the following procedure: 1) The attitude quaternion and gyroscope bias are set to their initial guesses where $\hat{\boldsymbol{q}}_{0 \mid-1} \in \mathbb{H}, \hat{\boldsymbol{b}}_{0 \mid-1} \in \mathbb{R}^{3}$ are the starting values of the attitude and gyroscope bias respectively. 2) The error estimates, $\boldsymbol{\delta} \boldsymbol{p}_{k \mid k}, \boldsymbol{\delta} \boldsymbol{\omega}_{k \mid k} \in \mathbb{R}^{3}$ of attitude and gyroscope bias respectively, are propagated in two different loops: 3) The gyroscope bias error is summed with the previous estimate of the gyroscope bias $\left(\hat{\boldsymbol{b}}_{k \mid k-1}\right)$ to produce the new estimate of the gyroscope bias $\left(\hat{\boldsymbol{b}}_{k \mid k}\right)$. 4) The attitude error is transformed back to an error quaternion using equation (20). 5) The integrated quaternion from the last iteration $\left(\hat{\boldsymbol{q}}_{k \mid k-1}\right)$ is rotated with the error by quaternion multiplication to produce the current estimate of the attitude while keeping the norm constraint.

This concludes the attitude part of the filter. 


\section{Position model}

1) States: The states for the translational estimation are chosen as:

$$
\hat{\boldsymbol{x}}_{k}^{P}=\left[\begin{array}{c}
\boldsymbol{p}_{k}^{F} \\
\boldsymbol{v}_{k}^{F}
\end{array}\right],
$$

where

$$
\begin{aligned}
& \boldsymbol{p}^{F}=\left[\begin{array}{lll}
p_{x}^{F} & p_{y}^{F} & p_{z}^{F}
\end{array}\right]^{T} \in \mathbb{R}^{3}, \\
& \boldsymbol{v}^{F}=\left[\begin{array}{lll}
v_{x}^{F} & v_{y}^{F} & v_{z}^{F}
\end{array}\right]^{T} \in \mathbb{R}^{3},
\end{aligned}
$$

are the position and velocity, both in the fixed frame respectively. Since the dynamics of the system is a double integrator from acceleration to the position the following system dynamics arise when using two $0^{\text {th }}$ order integrators, some times called a rectangle rules:

$$
\boldsymbol{f}\left(\hat{\boldsymbol{x}}_{k}^{P}, \boldsymbol{u}_{k}\right)=\left[\begin{array}{c}
\boldsymbol{p}_{k}^{F}+\Delta t \boldsymbol{v}_{k}^{F}+\frac{\Delta t^{2}}{2} \boldsymbol{a}_{k}^{F} \\
\boldsymbol{v}_{k}^{F}+\Delta t \boldsymbol{a}_{k}^{F}
\end{array}\right]
$$

where

$$
\boldsymbol{a}_{k}^{F}=\boldsymbol{g}\left(\hat{\boldsymbol{x}}_{k}, \boldsymbol{u}_{k}\right)
$$

is the application dependent acceleration model of the system.

2) Absolute measurements: The sensors that provide absolute measurements to the translational estimation is application dependent, but sensors such as GPS, camera sensor utilizing optical flow, height sensing sonar and barometer are a few popular ones. These however have a slow sampling rate compared to the rest of the filter which can be used to make the filter more computationally efficient by excluding the corresponding measurements from the prediction and measurement function when no new measurements are available.

This concludes the translational part of the filter.

\section{E. Parameter models}

Depending on the goals of the estimation parameter models could be added to estimate thrust or drag parameters for example, however these are all application specific. If parameter estimation is used the $\hat{\boldsymbol{x}}_{k}^{\theta}$ state vector is used to represent the estimated parameters.

\section{F. Complete system}

The complete system is the concatenation of the previous three subsystems as:

$$
\hat{\boldsymbol{x}}_{k}=\left[\begin{array}{c}
\hat{\boldsymbol{x}}_{k}^{A} \\
\hat{\boldsymbol{x}}_{k}^{P} \\
\hat{\boldsymbol{x}}_{k}^{\theta}
\end{array}\right] .
$$

The complete system can be used as a base for the estimation of attitude, position and parameters on any UAV by extending the acceleration and angular rate with the aircraft specific models. An example of this will be presented in the sequel where the estimation base is extended for the case of a quadrotor for attitude, position and parameter estimation.

\section{EXPERIMENTAL APPLICATION}

For the experimental application and evaluation of the proposed scheme, a quadrotor helicopter was chosen as the test platform, mainly due to their wide availability and possible range of applications. For the simulations static, dynamic and convergence simulations were performed and two experiments, one in attitude only mode and one in full estimation mode, were made to show the real world accuracy of the system.

In general, it is very difficult to ensure a consistency between the simulation and the experimental results. However, it should be noted that the aim of the simulations is not to act as a direct comparison with the experiments, but to extract the general characteristics of the estimation in a controlled simulation environment and in the sequel analyze the real world performance of the estimation scheme. In this article, an additional contribution is the fact that the proposed generalized estimation scheme has been also experimentally evaluated on a UAV, a realization that proves the applicability and the performance of the suggested scheme in real life and non controllable application scenarios.

\section{A. Application specific models}

Since the translational model is very dependent on the physics behind the aircraft, it is desirable to know how the control signal will affect position, velocity and acceleration. Any thrust generated by the engines in a multirotor platform will always point straight up, viewed from the body frame, while the sum of the forces can be calculated from [30], [31] as:

$$
F_{\text {tot }}=\sum_{i=1}^{n} F_{i}=A_{F} \omega_{\max }^{2} \sum_{i=1}^{n} u_{i}^{2}=A_{F} \omega_{\max }^{2} u_{e}
$$

where $F_{t o t}$ is the total thrust force generated, $A_{F} \in \mathbb{R}^{+}$is the thrust constant for the engines, $\omega_{\max } \in \mathbb{R}^{+}$is the maximum rotational rate of the engines, $i \in \mathbb{Z}^{+}$is the engine number, $u_{i} \in[0,1]$ is the control signal to each engine and $u_{e} \in \mathbb{R}^{+}$ is the sum of squared control signals. This can however be expanded further by using the known attitude and the mass of the aircraft to provide the application dependent acceleration model as:

$$
\begin{aligned}
\boldsymbol{g}\left(\hat{\boldsymbol{x}}_{k}, \boldsymbol{u}_{k}\right) & =\frac{F_{t o t}}{m} R(\hat{\boldsymbol{q}})^{T} \hat{\boldsymbol{e}}_{z}-\boldsymbol{g}^{F} \\
& =\beta u_{e} R(\hat{\boldsymbol{q}})^{T} \hat{\boldsymbol{e}}_{z}-\boldsymbol{g}^{F}
\end{aligned}
$$

where

$$
\beta=\frac{A_{F} \omega_{\max }^{2}}{m}
$$

is the mass compensated thrust coefficient. However, it takes a lot of measurements to find this parameter, plus $\beta$ varies with temperature, humidity, declining battery voltage and so on and thus in order to properly measure it and utilize it as a constant is an insufficient approximation. In contrast to this approach, we propose the more efficient approach to estimate it, a contribution that makes the overall filter to adapt to the current condition. The advantage of using such a mass compensation scheme is the fact that the thrust coefficient becomes directly observable from the accelerometer readings 
and since the estimation filter only needs the acceleration and the sum of squared control signals as input, the estimation becomes independent of the frame, both the number and geometrical location of engines, and mass. However, it is important to note that this acceleration model only works as long as drag has a minimal impact on the total acceleration.

However, this model still lacks dynamics and in our previous work [31] it has been depicted that a first order transfer function is a good approximation for the rotational speed of the motors. The problem here is that every motor/ESC combination will have its own unique time constant, hence for it to be compatible with the majority of motor/ESC combinations it needs to be estimated as well.

By assuming that the roll, pitch and yaw control signals are close to zero, the throttle input to thrust $\tilde{u}_{k}$ can be described as:

$$
\left[\begin{array}{c}
\tilde{u}_{k} \\
\tau_{k}
\end{array}\right]=\left[\begin{array}{c}
\frac{\tau_{k-1}}{\Delta t+\tau_{k-1}} \tilde{u}_{k-1}+\frac{\Delta t}{\Delta t+\tau_{k-1}} u_{k}^{t h r} \\
\tau_{k-1}
\end{array}\right],
$$

where $\tau_{k} \in \mathbb{R}^{+}$is the time constant of the motors and $u_{k}^{t h r} \in$ $\mathbb{R}^{+}$is the throttle control signal.

By combining this with the thrust model we get the following model for the acceleration, which includes the time constant of the motors, as:

$$
\left[\begin{array}{c}
a_{z}^{B} \\
\tilde{u}_{k} \\
\beta_{k} \\
\tau_{k}
\end{array}\right]=\left[\begin{array}{cc}
\beta_{k-1} \tilde{u}_{k}^{2} & \Delta t \\
\frac{\tau_{k-1}+\frac{\Delta t}{\Delta t+\tau_{k-1}}}{\Delta t+\tau_{k-1}} u_{k}^{t h r} \\
& \beta_{k-1} \\
\tau_{k-1}
\end{array}\right] .
$$

The GPS is used to provide a position fix in the horizontal plane and outputs longitude and latitude coordinates, which are transformed from the global Earth coordinate system to a local coordinate system using:

$$
\left[\begin{array}{l}
p_{x, m}^{F} \\
p_{y, m}^{F}
\end{array}\right]=\left[\begin{array}{c}
\left(\operatorname{lat}_{m}-\text { lat }_{r e f}\right) r_{\text {earth }} \\
-\left(\operatorname{lon}_{m}-\operatorname{lon}_{r e f}\right) \cos \left(\mathbf{l a t}_{m}\right) r_{\text {earth }}
\end{array}\right],
$$

where $\operatorname{lon}_{\text {ref }} \in \mathbb{R}$ and lat ref $\in \mathbb{R}$ are the starting, or "Home", position of the aircraft, $\operatorname{lon}_{m} \in \mathbb{R}$ and lat la $_{m} \in \mathbb{R}$ are the GPS measurements and $r_{\text {earth }} \in \mathbb{R}^{+}$is the radius of the Earth in meters. This is because 32-bit floating point arithmetic cannot represent the GPS coordinates without a loss of precision, which comes from the GPS coordinates having up to 9 significant digits where 32-bit floating point arithmetic can only represent 7 digits accurately, hence a local coordinate system is used to compensate for this drawback plus the starting position is always at zero in both $x$ and $y$ directions.

The height sensing Sonar measures ultrasound reflections from the ground, calculates the distance using time-of-flight and it is assumed that it has very narrow beam width. However, since in most of the cases the sonar is mounted to the bottom of the frame, the facing down and the rotation with the frame creates the need to have the measurements be compensated for allowing proper knowledge of the true height. This can be compensated by the attitude matrix where the $R_{3,3}$ element of the attitude matrix, in equation (12), contains the roll and pitch compensating cosines as presented in equation (14):

$$
R(\hat{\boldsymbol{q}})_{3,3} h_{\text {sonar }}=h_{\text {true }},
$$

where $h_{\text {sonar }} \in \mathbb{R}^{+}$is the measured height and $h_{\text {true }} \in \mathbb{R}$ is the true height. In practice the beam width of the sonar creates a dead zone, where the height measurement is the true height, with the same size as the beam width angle - this is however omitted in this implementation.

The Accelerometer measurements are made in the body frame of reference, with a bias from gravity and must be transformed into the fixed frame to be utilized in the estimation, while this is performed by using the attitude matrix as:

$$
\begin{aligned}
\boldsymbol{a}_{m, k}^{B} & =R(\hat{\boldsymbol{q}})\left(\boldsymbol{g}\left(\hat{\boldsymbol{x}}_{k}, \boldsymbol{u}_{k}\right)+\boldsymbol{g}^{F}\right) \\
& =\beta_{k} \tilde{u}_{k}^{2} \hat{\boldsymbol{e}}_{z},
\end{aligned}
$$

where $\boldsymbol{a}_{m}^{B} \in \mathbb{R}^{3}$ is the acceleration in the body frame and $\boldsymbol{g}^{F} \in \mathbb{R}^{3}$ is the gravitational offset in the sensor in the fixed frame of reference. Using these sensors the following state vector:

$$
\hat{\boldsymbol{x}}_{k}=\left[\begin{array}{llllll}
\boldsymbol{\delta} \boldsymbol{p}_{k}{ }^{T} & \boldsymbol{\delta} \boldsymbol{\omega}_{k}^{T} & \boldsymbol{p}_{k}^{F^{T}} & \boldsymbol{v}_{k}^{F^{T}} & \beta_{k} & \tau_{k}
\end{array}\right]^{T}
$$

and the following measurement vector arises:

$$
\boldsymbol{z}_{k}=\left[\begin{array}{lllll}
\boldsymbol{\theta}_{e, k}^{T} & p_{x, m, k}^{F} & p_{y, m, k}^{F} & h_{m, k}^{B} & \boldsymbol{a}_{m, k}^{B}{ }^{T}
\end{array}\right]^{T},
$$

which makes the measurement prediction function as

$$
\boldsymbol{h}\left(\hat{\boldsymbol{x}}_{k \mid k-1}\right)=\left[\begin{array}{c}
R\left(\hat{\boldsymbol{q}}_{k \mid k-1}\right)^{T} R\left(\boldsymbol{\delta}_{k \mid k-1}\right)^{T} \boldsymbol{\delta} \boldsymbol{p}_{k \mid k-1} \\
p_{x, k \mid k-1}^{F} \\
p_{y, k \mid k-1}^{F} \\
\frac{p_{z, k \mid k-1}^{F}}{R\left(\hat{\boldsymbol{q}}_{k \mid k-1}\right)_{3,3}} \\
\beta_{k} \tilde{u}_{k}^{2} \hat{\boldsymbol{e}}_{z}
\end{array}\right] .
$$

However, these measurements are being sampled at different rates: normal GPS rates are $1-10 \mathrm{~Hz}$, Sonar rates are 10 - $20 \mathrm{~Hz}$ and the Accelerometer rates are $1 \mathrm{~Hz}-8 \mathrm{kHz}$. In this system the Accelerometer rate has been set to 200 $\mathrm{Hz}$ and during all the samples, where no new information has entered the system from any of the other sensors, the corresponding acceleration measurements are removed from the measurement vector and measurement prediction function. In this approach the covariances will propagate correctly when there are no measurements and in the majority of iterations the measurement vector will be of size 6 with a worst case size of 9 , that comes from the error angles (size 3), accelerometer (size 3), GPS (size 2) and sonar (size 1), which greatly reduces the computational complexity of the filter.

\section{B. Simulations}

The simulations were performed for three cases: a) when the system is stationary and converged to show the stationary accuracy, b) when offsets and biases are applied to evaluate the overall convergence of the suggested scheme, and c) in a dynamical scenario, when the system is accelerating 
from standstill and then decelerating to a complete stop, with both small and large accelerations, to show the dynamical performance and accuracy.

The simulations were carried out with a magnetometer sampling of $75 \mathrm{~Hz}$ and with a standard deviation $\sigma_{m}=10^{-3}$ Gauss, gyroscope sampling of $200 \mathrm{~Hz}$ with a standard deviation $\sigma_{\omega}=0.1 \mathrm{rad} / \mathrm{s}$, accelerometer sampling of $200 \mathrm{~Hz}$ with a standard deviation $\sigma_{a}=0.3 \mathrm{~m} / \mathrm{s}^{2}$, sonar sampling of $10 \mathrm{~Hz}$ with a standard deviation $\sigma_{s}=10^{-2} \mathrm{~m}$ and a GPS sampling of $5 \mathrm{~Hz}$ with a standard deviation $\sigma_{G P S}=1 \mathrm{~m}$.

1) Stationary simulations: In the first case of stationary simulations, as presented in Figure 4, it is assumed that the estimated parameters have converged and the system is at rest, implying hovering without disturbances. The system is very accurate with an RMSE of 6.4 millirad in pitch and roll and 25.3 millirad in yaw. The decrease of accuracy in yaw comes from the fact that the magnetometer only measures a small part of the desired vector - reducing the useful information in the measurement.

The estimation of angular rate is only using bias compensation from the measurement, where the noise has the same amplitude as in the measurement but with a zero mean. Post processing of the angular rate can be made to reduce the noise, however this was not included in this implementation.

The velocity estimation showed very good results at hover with an RMSE error of $0.11 \mathrm{~m} / \mathrm{s}$ in $x$ and $y$ and $0.086 \mathrm{~m} / \mathrm{s}$ in $z$. The result is showing some signs of drift, however the drift is bounded by the GPS measurements and if greater accuracy is needed in velocity, a sensor measuring the velocity is needed.

When it comes to the estimation of position the result is very good compared to the GPS measurements. The position has an RMSE error of $0.24 \mathrm{~m}$ in $x$ and $y$ and $0.055 \mathrm{~m}$ in $z$. As with velocity it is showing signs of drift however the position is bounded within $0.5 \mathrm{~m}$. This comes from the drift of the velocity since the position is integrated from the velocity with only small corrections from the GPS, however this is enough to keep the drift bounded and close to zero. The position estimation of height has much higher accuracy since the sonar measures the height with much lower noise.

2) Convergence simulations: The second case examined is how well the system finds errors in the sensors, such as biases and constants, and system parameters, such as the thrust constant, as depicted in Figure 5. The gyroscope bias was set to $0.1,0.05$ and $-0.1 \mathrm{rad} / \mathrm{s}$ for the roll pitch and yaw respectively and with a starting guess of $0 \mathrm{rad} / \mathrm{s}$ and the starting guess for $\beta$ was set to $15 \mathrm{~m} / \mathrm{s}^{2}$ with the true at $9.8 \mathrm{~m} / \mathrm{s}^{2}$. As it can be seen in Figure 5's subplots, all the estimated parameters converge quickly with sings of exponential convergence, without offset and with very low noise, except the yaw bias, which first diverges. This is however expected since the pitch and roll must first converge before the yaw has a correct reference frame. These are essential for the stability of the system, since these parameters define the physical properties of the sensors and the system - hence in a real implementation, a lot of care should be taken to present the estimation with a good initial guess.

Due to the complexity of getting a good convergence of the motors' time constant during simulation, when there are almost
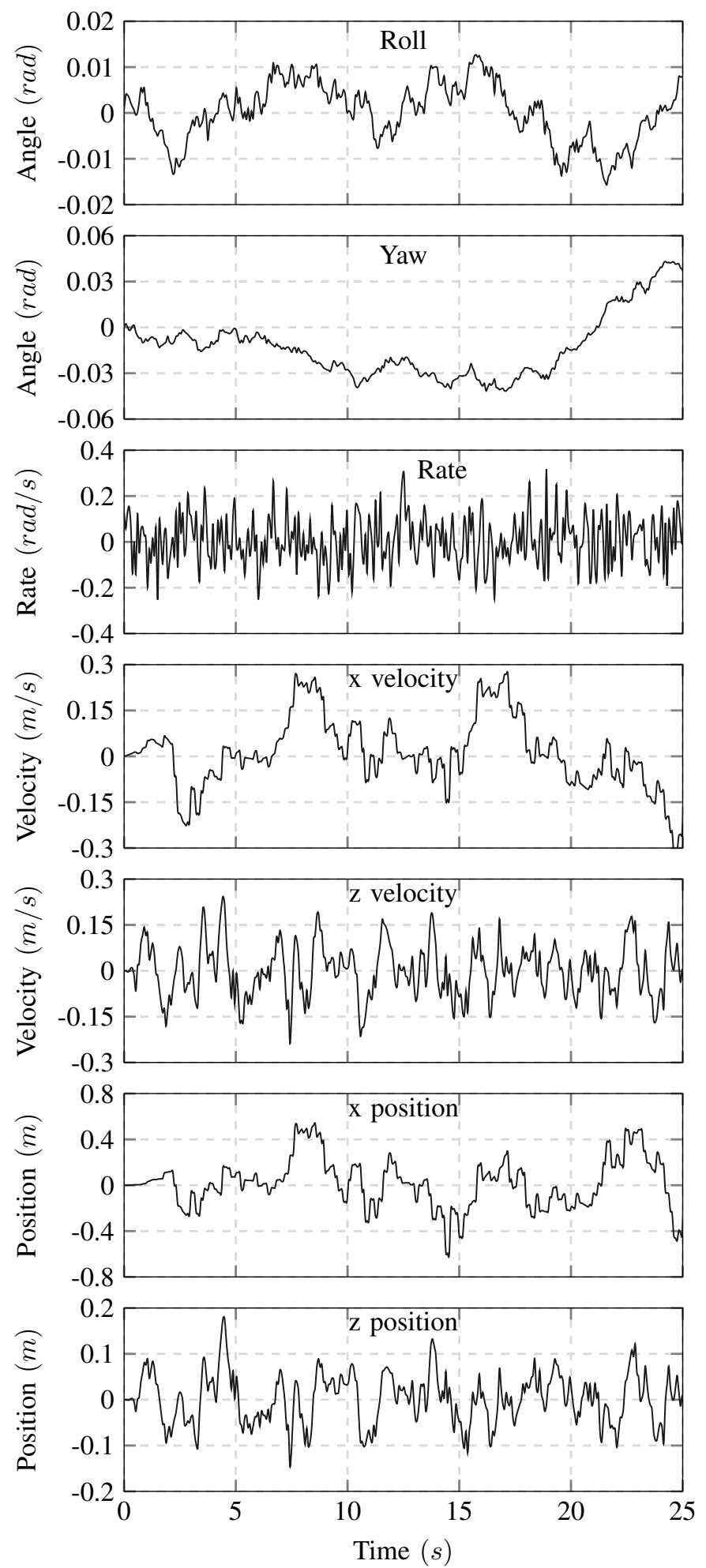

Fig. 4. The first two plots show estimation results of roll and yaw when stationary. The results are showed in radians for a more intuitive presentation. The result of pitch has the same characteristics as that of the roll and has been omitted. The third shows the measured angular rate around roll with bias compensation. The result of angular rate around pitch and yaw has the same characteristics as that of the roll and has been omitted. The fourth and fifth shows the estimated $x$ and $z$ velocities in the fixed frame of reference. The result of $y$ velocity has the same characteristics as that of the $x$ velocity and has been omitted. While the sixth and the seventh shows the estimated $x$ and $z$ position in the fixed frame of reference. The result of $y$ position has the same characteristics as that of the $x$ position and has been omitted. 

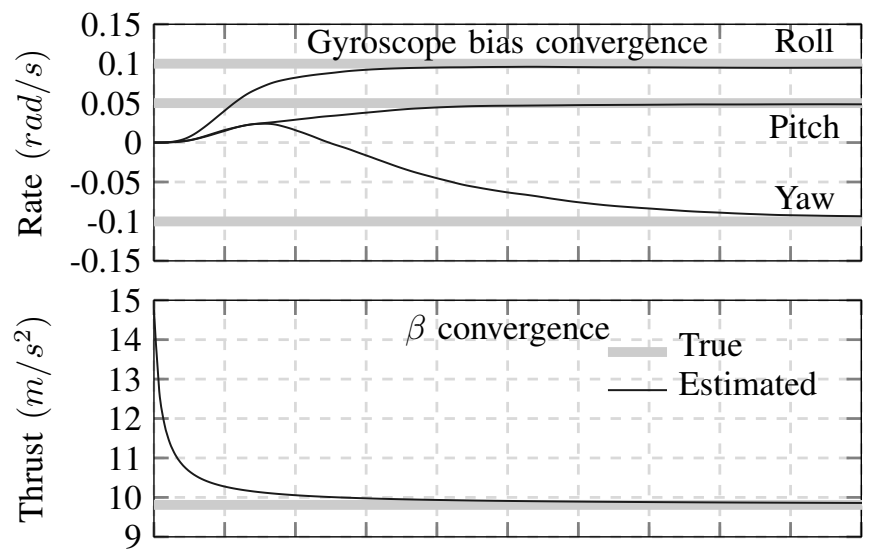

Time $(s)$

Fig. 5. A complete overview of the convergence of estimated parameters where the first plot denotes the gyroscope bias and the second is the mass compensated thrust parameter.

no excitation, these has been left out to the real experiment where ample excitation exists.

3) Dynamical simulations: The last case examined was the tracking performance of the estimator, which was evaluated on a simple roll maneuver, depicted in Figure 6, designed to accelerate and decelerate the aircraft. During the roll of
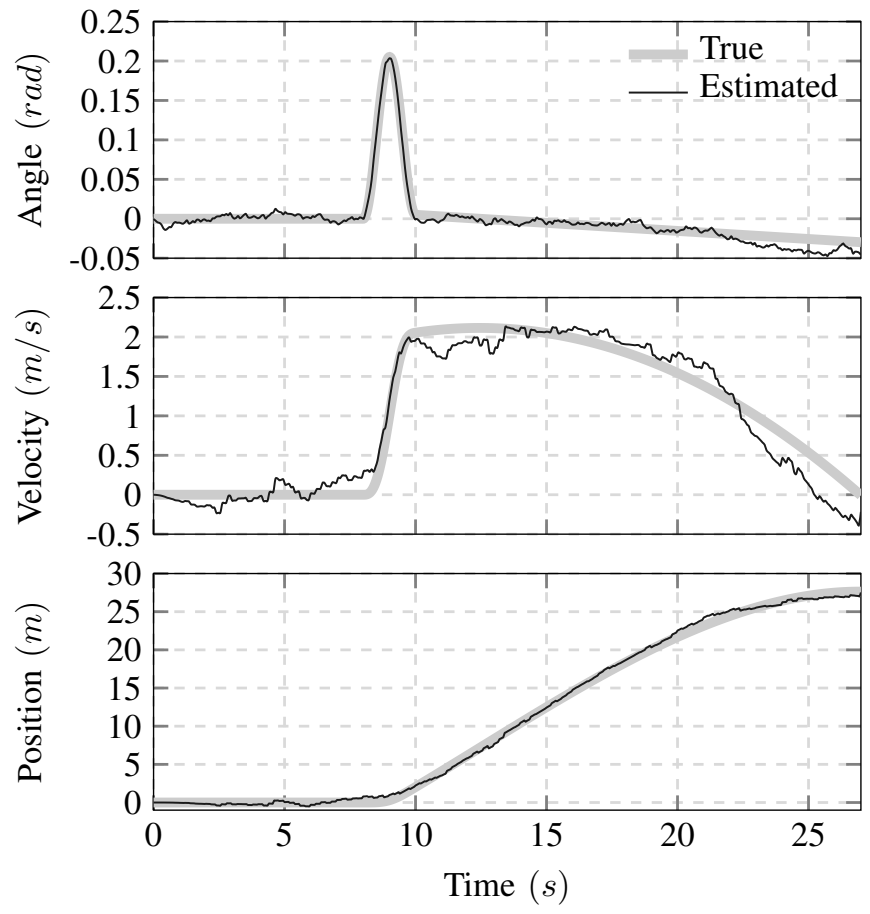

Fig. 6. The first plot shows the roll angle during dynamic movements and the result is presented in radians for intuitive display. The second shows the velocity during dynamic movements. And the third shows the position during dynamic movements. The solid thin line is the estimate and the thick gray line is the true value.

the aircraft the attitude estimation tracks very well with small errors and no signs of a constant offset, since the integration of the quaternion provide relative attitude updates.

The velocity tracking is the one with the most error, since it isn't directly observed via measurements. However, the proposed scheme is still able to track well and does not show signs of divergence nor offset. It should be noted that since the model to predict accelerations, from Section IV-A, gives very accurate results during fast changes until the system stops tilting, which is the case when noise will have a major part of the resulting velocity.

Finally the tracking of position is very good, with small errors and no offsets. This shows that the cross coupling from the attitude part works as intended, since it can utilize the acceleration to predict the velocity and position accurately, plus the pressure constant and thrust parameter does not diverge during the maneuver.

4) Complexity: The effect of adding more measurements compared to the naive case of either using infinite covariance for the measurements, which have no new information or repeating the old input and increasing the measurement covariance accordingly is presented in Table I. For a complete computational complexity comparison with other implementations, a generic comparison with a SR-UKF, as presented in [32], and and SR-CKF, as presented in [11], was made, as depicted in Table II. The comparison was made in the best case and worst case, which is when the measurement vector is of size 6 and 9 respectively, over 100000 iterations and the results were compared to the fastest of each considered case to present relative differences. In the comparison, the SR-EKF implementation was significantly faster than the SR-UKF and the SR-CKF and this gain comes mainly from the fact that the SR-UKF has two extra Cholesky updates that the SRCKF and SR-EKF avoids, while the SR-EKF does not need to evaluate the state prediction and measurement prediction functions multiple times as the SR-UKF and SR-CKF needs to do. The comparisons between the filters were made on an Intel Core i7-4770 CPU running at $3.4 \mathrm{GHz}$ with 16 GByte of RAM, and the implementations of all the filters with the corresponding comparisons, in Matlab, are available online at [33]. The RMSE of position is calculated as:

$$
\text { Position RMSE }=\sqrt{\operatorname{RMSE}_{x}^{2}+\operatorname{RMSE}_{y}^{2}+\mathrm{RMSE}_{z}^{2}} \text {. }
$$

The RMSE error was selected since this is the most common approach in the bibliography to present the errors independently of their sign and magnitude. The comparisons were made in the dynamical test and during this comparison the GPS rate was set to $5 \mathrm{~Hz}$ and the Sonar rate was set to $20 \mathrm{~Hz}$.

When adding more sensors, with low sampling rates, the overall computational complexity does not increase as much as when using the naive approach, however the RMSE decreases rapidly. This clearly shows the effect of removing measurements that does not contain any new information, as discussed at the end of Section III-D2.

It should also be noted that since the measurements have different sampling intervals, the chance that two or more measurements come in at the same time is very low, making the case with all sensors almost the same as adding the contributions from each sensor.

\section{Experimental verification}

For the experimental verification of the proposed estimation scheme a motion capture system, consisting of 20 Vicon T40s 
TABLE I

COMPARISON OF COMPUTATIONAL COMPLEXITY WHEN USING DIFFERENT CONFIGURATIONS OF SENSORS DURING THE DYNAMICAL TEST.

\begin{tabular}{lcl} 
Sensors & Normalized calculation time & Position RMSE \\
\hline Base* & 1.00 & $5.431 \mathrm{~m}$ \\
Base + GPS & 1.09 & $0.8989 \mathrm{~m}$ \\
Base + Sonar & 1.14 & $4.133 \mathrm{~m}$ \\
All & 1.26 & $0.4149 \mathrm{~m}$ \\
Naive (All) & 4.68 & $0.4149 \mathrm{~m}$ \\
\hline
\end{tabular}

* Reference Vector, Accelerometer, Gyroscope and Magnetometer

TABLE II

COMPARISON OF COMPUTATIONAL COMPLEXITY BETWEEN THE SR-UKF, SR-CKF AND THE SR-EKF OVER 100000 ITERATIONS

\begin{tabular}{lcccc} 
Filters & Best case & Worst case & Relative best & Relative worst \\
\hline SR-UKF & $44.8 \mathrm{~s}$ & $44.5 \mathrm{~s}$ & 3.88 & 3.42 \\
SR-CKF & $35.7 \mathrm{~s}$ & $36.0 \mathrm{~s}$ & 3.09 & 2.77 \\
SR-EKF & $11.6 \mathrm{~s}$ & $13.0 \mathrm{~s}$ & 1 & 1 \\
\hline
\end{tabular}

cameras were utilized to capture the true data for comparison with the estimated data. This was chosen due to the fact that the motion capture system has very high accuracy and does not drift in accuracy nor time making it perfect to use as a global reference. The camera system captures the true pose (attitude: roll, pitch, yaw and position: $x, y, z$ ) at $200 \mathrm{~Hz}$.

\section{Experimental setup}

1) Electronic hardware: The data acquisition and control is handled from a system called the KFly designed and developed at Luleå University of Technology, with an overview of the system architecture presented in Figure 7.

Micro Controller Unit: The MCU driving the system is ST's STM32F405RGT6, an 32-bit ARM Cortex-M4F CPU featuring a DSP core and an 32-bit single precision FPU, all running at $168 \mathrm{MHz}$. It has two built in 32-bit timers and twelve 16-bit timers each with four PWM outputs. For offloading the MCU from data transfer tasks it features a 16channel DMA controller with Memory to Memory, Peripheral to Memory and Memory to Peripheral transfer modes.

Sensors: The system uses three main sensor chips on board mainly used for estimation and control:

- Invensense's MPU-6050 Accelerometer and Gyroscope SOC for measuring acceleration and angular velocity, with a full scale range of $\pm 16 \mathrm{~g}$ and \pm 2000 degrees/s, both with 16-bit accuracy. It is sampling at $200 \mathrm{~Hz}$ and running each sample trough an internal low-pass filter with an $44 \mathrm{~Hz}$ cut-off frequency. Both the accelerometer and the gyroscope has resonance frequencies well outside (above $30 \mathrm{kHz}$ ) the vibrations created by an air frame. The sensor communicates with the $\mathrm{MCU}$ via an $\mathrm{I}^{2} \mathrm{C}$ bus running at $400 \mathrm{kbit} / \mathrm{s}$.

- Honeywell's HMC5983 Magnetometer for detecting North/South/East/West with 5 milli-Gauss resolution. Sampling is done at $75 \mathrm{~Hz}$ and it uses the same $\mathrm{I}^{2} \mathrm{C}$ bus as the previous sensor.

- MEAS Switzerland's MS5611-01BA03 Barometric Pressure Sensor used as an altimeter utilizing a 24-bit $\Delta \sum$ ADC for an altitude resolution of $10 \mathrm{~cm}$. Sampling is done at $10 \mathrm{~Hz}$ and it uses the same $\mathrm{I}^{2} \mathrm{C}$ bus as the previous sensors. Hence all sensors share the same communication bus as depicted in Figure 7.

Communication: The system has four expansion ports, each featuring a UART running at a maximum of 7.2 Mbaud, a single CAN port and a USB configured as a VCP for increased flexibility. All ports have protection from ESD and over current protection. Wireless communication such as X-Bee, Zig-Bee or Bluetooth or additional sensors such as GPS, optical flow or sonar can be connected to the UART ports to expand its capabilities.

Operating System: The system features the ChibiOS/RT kernel [34]. ChibiOS handles the communication with the hardware, the mutual exclusions for the communication buses, ISR handler synchronization and data processing synchronization.

2) Attitude cross: To evaluate the attitude tracking of the system presented in Figure 2, a special hand held cross, inspired from [35], was built to be able to placing tracking markers at the end of each beam. This cross, depicted in Figure 8, can then be manually tilted for evaluate the attitude convergence of the system under controlled circumstances.

3) Quadrotor frame: The quadrotor frame used in the experiments, depicted in Figure 9, was designed and manufactured at Luleå University of Technology, with the goal of being a low vibration frame in order to minimize the contamination of sensor data from noise. Hence the frame is of a two part design, where the bottom part, holding the motors and ESCs, is separated from the other part, which is holding the battery and sensors, with silicone dampeners. The battery, in conjunction with the silicone dampeners, creates a mechanical low-pass filter drastically reducing the measured noise in the sensors.

The motors used on the frame are the Tiger Motor's MN1804 of the navigator series, the ESCs are Tiger Motor's T12A ESC and the propellers used are Tiger Motor's 6x2 inch Carbon Fiber propellers. For receiving RC-inputs an FR-Sky receiver, the D4R-II, was used and to stream the sensor data for logging an X-Bee Pro S1 was used at 115200 baud with the "two stop bit"-fix for high data rate transfers.

However, even at the maximum transfer speed, all data could not be transmitted due to the massive amounts of data generated by the sensors and hence the choice was made to drop the barometer measurements.

\section{E. Experiment: Attitude only}

To test the performance of the error-based attitude estimation, the attitude cross in Figure 8 was used. The experiment was designed to do three simple movements in each roll, pitch and yaw axis captured by both the internal sensors and the external motion capture system. The reference vector used in the experiment is the gravitational vector measured by the accelerometer.

The result of the experiment, depicted in Figure 10, shows that the system converges quickly and tracks dynamic changes very well. As the system is based on integration of the gyroscope and the error estimation only corrects the integration this is what is expected. However, in both pitch and roll, there are 


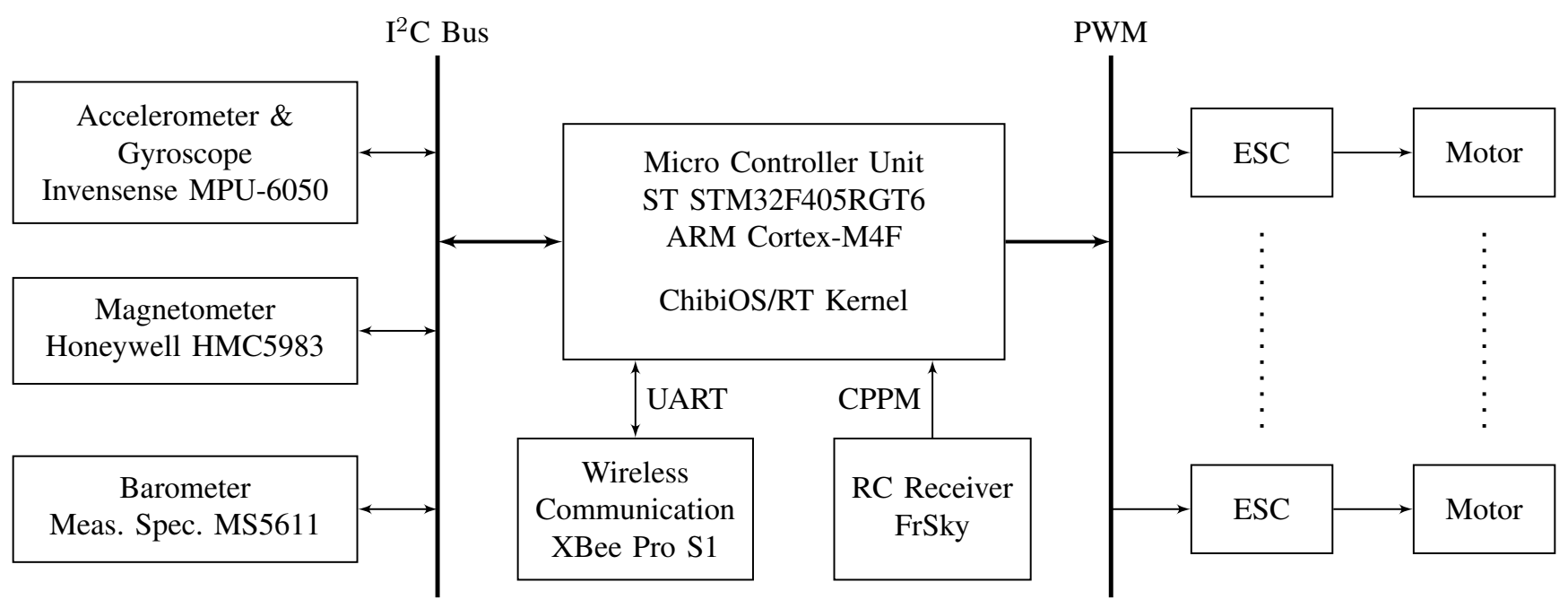

Fig. 7. A detailed overview of the hardware architecture of the KFly system.

moments of divergence which comes from the positioning of the inertial sensors to the rotational center. The accelerometer picks up the centripetal accelerations which comes in as offsets in the estimation. This is most evident during the yaw motion, when the distance to the rotational center is at its maximum.

In the motion capture room there were a lot of disturbing magnetic fields which made the magnetometers measurements less useful as can be seen in the yaw estimation as it diverges slightly. The movement during the yaw motion does sample the magnetic field enough to get a good estimation of the gyroscope's bias and get complete convergence which is quite evident in the yaw bias estimation.

\section{F. Experiment: Full estimation}

To evaluate the full proposed estimation scheme, the quadrotor depicted in Figure 9 was flown manually inside the motion

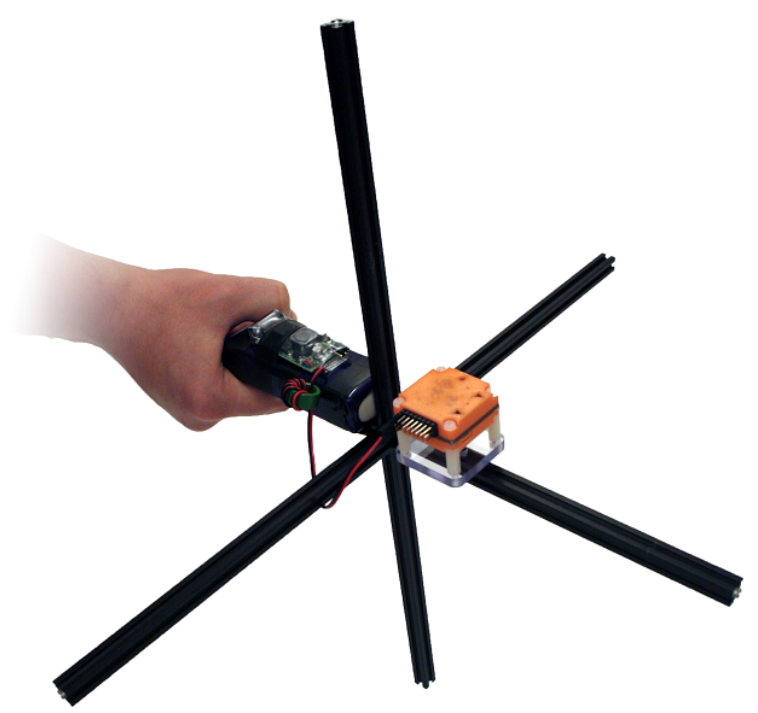

Fig. 8. A photo depicting the KFly system attached to the hand-held attitude cross. In this photo the tracking markers for the cameras and the $\mathrm{RF}$ communication have been omitted.

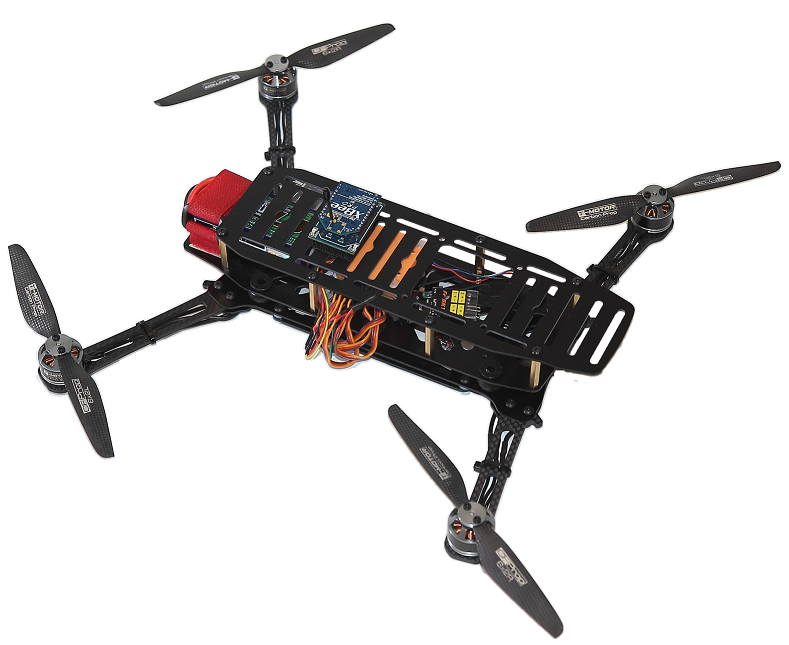

Fig. 9. A photo of the vibration damped quadrotor frame used in the experiments. In the state depicted it is ready to fly with all necessary components attached. In this photo the tracking markers for the cameras have been omitted.

capture arena to capture the full 6-DOF (roll, pitch, yaw and $x, y, z$ position), while simultaneously streaming the inertial data generated from the internal sensors over an XBee RF-link, as depicted in Figure 7. The problem with flying inside is that there is no GPS reception and to counter this problem GPS drift was measured outside by placing the GPS in "pedestrian"-mode to allow it to drift freely, with a uBlox NEO-M8 GPS, as depicted in Figure 11, and was later added to the camera measurements to simulate a GPS. The conversion from longitude and latitude to local error coordinates was made using equation (38).

Finally the data from the flight was logged to do two different estimations on the same data, a) use the cameras to provide the general attitude reference vector and $b$ ) to use the accelerometer as an non-ideal reference. This difference is depicted in Figure 12 and 13 by the solid and dashed lines, where the solid line, named "Estimation", is using the 

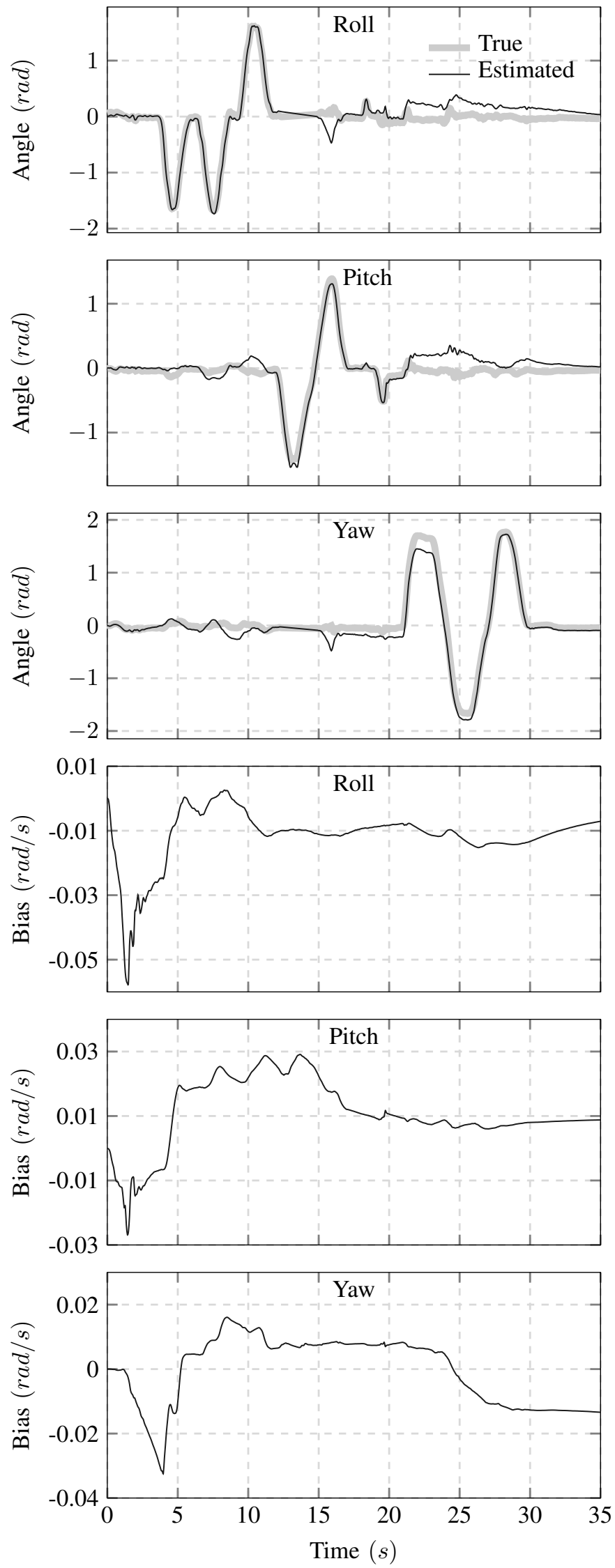

Fig. 10. Estimation results from the "attitude only"-estimation on the hand held cross. The first three sub-figures shows the attitude convergence in roll, pitch and yaw respectively whilst the last three sub-figures shows the gyroscopes' bias convergence in roll, pitch and yaw respectively.
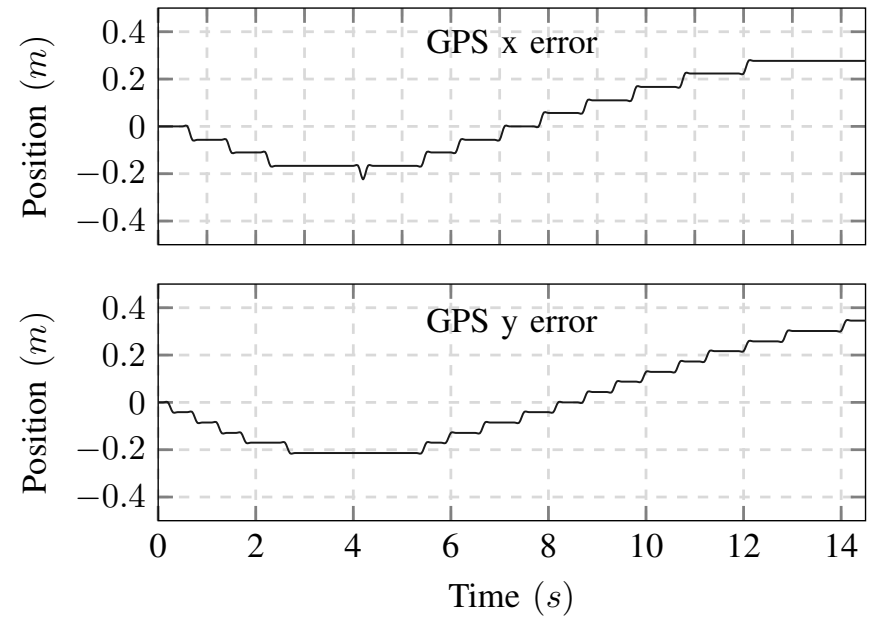

Fig. 11. Measured GPS drift using an uBlox NEO-M8 GPS, converted to local coordinates using equation (38), to be added to the camera measurements for simulating a GPS indoors.

accelerometer as reference and the dashed line, named "Ideal", is using the camera generated reference vector. All position measurements are made at $10 \mathrm{~Hz}$.

The other difference between the two cases is that during the "Ideal" case the system is not given a valid guess for the gyroscopes' biases to show convergence during flight, while in the in the "Estimation" case the biases were measured before take-off and used as the initial guess for the estimation to have a more realistic usage case.

Position estimation: The position estimation is analyzed as two cases, a) the horizontal estimation and b) the vertical estimation, as the horizontal estimation receives most effect from the estimated angles, while the vertical estimation receives most effect from the estimated acceleration, including the trust parameters.

The horizontal estimation, when compared between the ideal and fully estimated case, receives most of its error from the error in the GPS, which is expected as it is the only reference in the system. However as the acceleration, estimated from the tilting of the aircraft, is integrated twice, small errors will make the position estimation diverge. It can be clearly seen when the GPS measurements corrects the position estimation as jagged noise on the peaks of the position estimation.

The vertical estimation relies heavily on the parameter estimation to be correct and estimate the acceleration properly, and the $z$-axis acceleration, depicted in the last sub-figure in Figure 13, converges with very small errors helping the vertical estimation converge. Even though there are only 10 measurements per second the double integrated acceleration provides the dynamic response needed to get highly accurate estimation.

Attitude \& bias estimation: The pitch and roll estimation in the ideal case starts with relatively high errors until the bias estimation converges at about 6-8 seconds. After this convergence, the attitude estimation tracks the response of the aircraft with very small errors. However the gyroscopes' biases are moving quite a lot, while this effect is produced mainly by the gains of the gyroscopes not being exactly as 

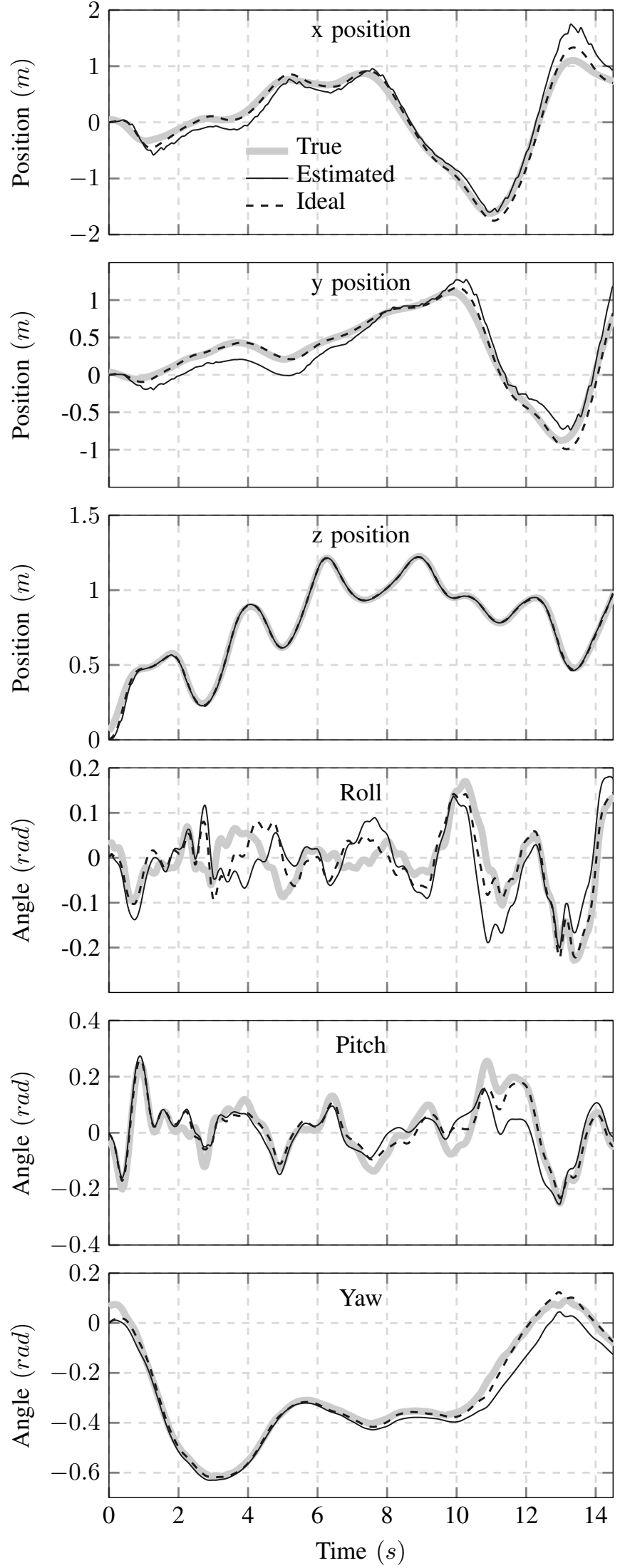

Fig. 12. Estimated versus camera measured position and attitude. The first three sub-figures shows $x, y$ and $z$ position respectively and the last three sub-figures show roll, pitch and yaw respectively. The thick gray line is the camera reference, dashed line is the estimation based of an ideal attitude vector and the solid line is the true full estimation based of inertial sensors. specified, so the biases compensate this error in the gyroscopes as well. Overall, the estimation based of the accelerometer shows, as expected, less accuracy than when using an ideal attitude reference.

The yaw estimation is, in the ideal case, tracking very well, however in the case where the magnetometer is used, there is a constant offset from the erroneous magnetic fields inside the flying area. Great caution was taken so that the motors and ESCs did not affect the magnetometer, but changes in the magnetic field due to the building could not be removed more than general and frequent calibrations inside the motion capturing room. With this said, the yaw estimation is working as expected as its errors have no significant effect such as adding a constant offset to the roll and pitch estimations.

Parameter estimation: As there is no ground truth for the thrust parameter and the time constant of the motors, the only thing that tells how well the estimation is working is to compare the estimated acceleration from the parameter estimation and throttle to the measured acceleration as done in the last sub-figure in Figure 13. From the obtained results, it is straight forward that the proposed scheme works very well and has almost no error when negating the noise from the frame. What makes the acceleration look like it is taking a multitude of steps comes from the fact that the streamed throttle signal is quantized due to the restrictions of the available RF bandwidth and hence it takes steps of $1 \%$.

Also to be noted is that both the ideal and full estimation estimates the same parameters. This comes from the parameter estimation being observable through the accelerometer measurements and being decoupled from the attitude and position of the UAV.

\section{DISCUSSION}

One of the main points of this article has been how to reduce the computational complexity of the algorithms for implementation in small embedded systems and this can further be expanded by analyzing the prediction models and measurement equations. One main point is since the error states are zeroed, after each iteration, the rotation matrices based on the error states, in equations (27-29), become identity matrices and the derivative of the GRP from equation (21) becomes zero - both simplifying the implementation.

Since the choice was made to use the square-root version of the EKF, numerical precision and dynamical range are effectively doubled, a fact that allows for implementation in small embedded systems without the risk of problems with the parameters. As an added bonus the covariance matrix of the SR-EKF is guaranteed to be a positive-definite matrix and if any update is about to break that constraint the Cholesky down date will get a $\sqrt{-1}$ in its calculations and report an error which can be handled.

One of the contributions of the proposed integrated estimation scheme for the multirotors field is the estimation of the mass compensated thrust coefficient $\beta$ and the time constant $\tau$ of the motors. This allows the system to be used in any configuration of multirotors, whilst providing valuable information about the thrust, and its response, available to the 

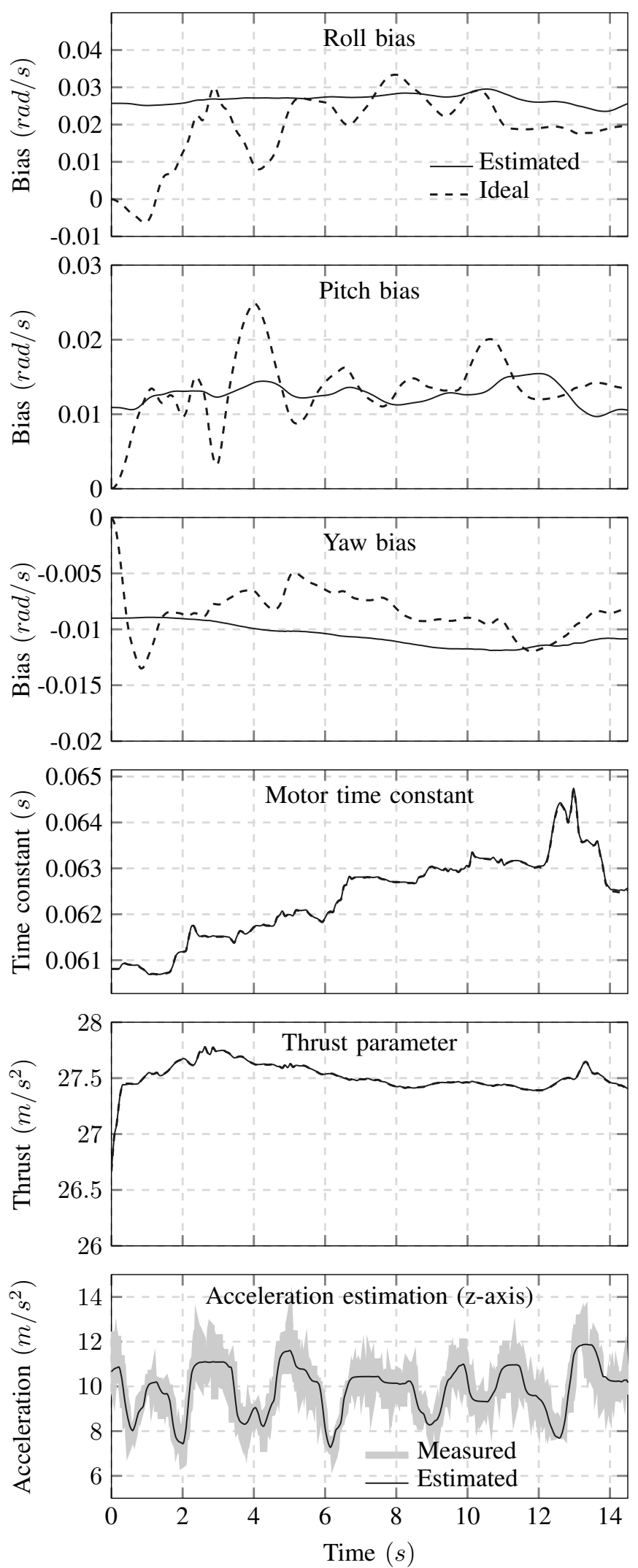

Fig. 13. Estimated system parameters. The first three sub-figures show roll, pitch and yaw bias respectively, the next two sub-figures show the motor time constant and thrust parameter respectively and the last sub-figure show the comparison of measured acceleration versus estimated acceleration. The thick gray line is measured acceleration, dashed line is the estimation based of an ideal attitude vector and the solid line is the true full estimation based of inertial sensors. controller. This information can be utilized further as a feed forward term to provide the throttle needed for hovering and to predict accelerations caused by tilting, plus the time constant can be used to tune the controller for online auto-tuning.

It should be noted that it has been assumed that the starting guess of the attitude filter is good as it uses approximations that rely on the errors to be close to zero. Such an approach is generic and connected to the reality, since the attitude can be measured during the start up phase, which will provide small starting errors. Acceptable start up errors are: a) the sign of the vector projections in equation (26) to be correct, thus allowing an error less than $\pm \pi / 2$ radians, and b) the values should not be plus nor minus infinity.

Finally, it should be mentioned that the assumption made for the time constant of the thrust and the thrust constant being much slower than the sampling time of the estimator is confirmed by the experiments, where the thrust constant is practically a constant and the time constant is approximately 12 times smaller than the sampling time. An additional theoretical analysis of the effect of this assumption is considered as future work.

\section{Conclusions}

The aim of this work was to develop and experimentally evaluate a generalized approach to position, attitude and parameter adaptive estimation based on error parameters. The suggested scheme utilizes a square-root formulation of the Extended Kalman Filter to guarantee the semi-positive definiteness of the covariance matrices and to counter the problem of dynamical range in small embedded systems by effectively doubling the dynamical range of the calculations. The proposed approach counters the problem of computational complexity by utilizing error based states and different sampling rates on different sensors that have a direct effect in reducing the total computational complexity. The presented work had the following significant merits: a) The generalized approach made the estimation applicable in any kind of UAV, where only the vehicle dependence needed to be added, with minimal computational impact, b) the approach allowed the addition of parameter adaptive estimation as showing in the case of a quadrotor, where parameters for the trust and time constants were found online to improve the estimation, and c) the use of projections to bound the errors, introduced in the attitude estimation by stray magnetic fields, where the errors were bounded to the yaw only.

\section{REFERENCES}

[1] K. Alexis, G. Nikolakopoulos, A. Tzes, and L. Dritsas, "Coordination of helicopter UAVs for aerial Forest-Fire surveillance," in Applications of Intelligent Control to Engineering Systems. Springer Netherlands, June 2009, pp. 169-193.

[2] M. A. Goodrich, J. L. Cooper, J. A. Adams, C. Humphrey, R. Zeeman, and B. G. Buss, "Using a Mini-UAV to Support Wilderness Search and Rescue Practices for Human-Robot Teaming," in Proceedings of the IEEE International Conference on Safety, Security and Rescue Robotics, Rome, Italy, September 2007. [Online]. Available: http://www.cs.utexas.edu/users/ai-lab/?goodrich:ssrr07

[3] J. Fink, N. Michael, S. Kim, and V. Kumar, "Planning and control for cooperative manipulation and transportation with aerial robots," The International Journal of Robotics Research, vol. 30, no. 3, March 2011. [Online]. Available: http://ijr.sagepub.com/content/early/2010/09/21/0278364910382803 
[4] E. Fresk and G. Nikolakopoulos, "Full Quaternion Based Attitude Control for a Quadrotor," European Control Conference, 2013.

[5] G. Raffo, M. Ortega, and F. Rubio, "An integral predictive/nonlinear control structure for a quadrotor helicopter," Automatica, vol. 46, no. 1, pp. 29-39, 2010.

[6] K. Alexis, G. Nikolakopoulos, and A. Tzes, "Switching model predictive attitude control for a quadrotor helicopter subject to atmospheric disturbances," Control Engineering Practice, vol. 19, no. 10, pp. 1995-1207, October 2011.

[7] H. Lim, J. Park, D. Lee, and H. Kim, "Build Your Own Quadrotor: Open-Source Projects on Unmanned Aerial Vehicles," IEEE Robotics \& Automation Magazine, vol. 19, p. 33.45, 2012.

[8] F. L. Markley, "Attitude Error Representations for Kalman Filtering," Journal of Guidance, Control, and Dynamics, vol. 26, pp. 311-317, 2003.

[9] J. L. Crassidis and F. L. Markley, "Unscented Filtering for Spacecraft Attitude Estimation," Journal of Guidance, Control, and Dynamics, vol. 26, pp. 536-542, 2003.

[10] Y.-J. Cheon and J.-H. Kim, "Unscented Filtering in a Unit Quaternion Space for Spacecraft Attitude Estimation," IEEE International Symposium on Industrial Electronics, pp. 66-71, 2007.

[11] I. Arasaratnam and I. Simon Haykin, Fellow, "Cubature Kalman Filters," IEEE Transactions on Automatic Control, vol. 54, pp. 1254 - 1269, 2009.

[12] X. Tang, J. Wei, and K. Chen, "Square-Root Adaptive Cubature Kalman Filter with Application to Spacecraft Attitude Estimation," International Conference on Information Fusion, pp. 1406-1412, 2012.

[13] M. Ahmadi, S. Shiraz Univ., A. Khayatian, and P. Karimaghaee, "Orientation Estimation by Error-State Extended Kalman Filter in Quaternion vector space," SICE Annual Conference, pp. 60-67, 2007.

[14] N. Trawny and S. I. Roumeliotis, "Indirect Kalman Filter for 3D Attitude Estimation," Department of Computer Science \& Engineering, Tech. Rep., 2005.

[15] I. Arasaratnam and S. Haykin, "Square-Root Quadrature Kalman Filtering," IEEE Transactions on Signal Processing, 2008.

[16] R. Mahony, T. Hamel, and J.-M. Pflimlin, "Nonlinear complementary filters on the special orthogonal group," Automatic Control, IEEE Transactions on, vol. 53, no. 5, pp. 1203-1218, 2008.

[17] S. Bonnabel, P. Martin, and P. Rouchon, "Non-linear symmetrypreserving observers on Lie groups," Automatic Control, IEEE Transactions on, vol. 54, no. 7, pp. 1709-1713, 2009.

[18] R. C. Leishman, J. C. Macdonald, R. W. Beard, and T. W. McLain, "Quadrotors and accelerometers: State estimation with an improved dynamic model," Control Systems, IEEE, vol. 34, no. 1, pp. 28-41, 2014.

[19] D. B. Kingston and A. W. Beard, "Real-time attitude and position estimation for small UAVs using low-cost sensors," in AIAA 3rd Unmanned Unlimited Technical Conference, Workshop and Exhibit, 2004.

[20] R. Mahony, V. Kumar, and P. Corke, "Multirotor aerial vehicles: Modeling, estimation and control of quadrotor," IEEE Robotics and Automation Magazine, vol. 19, no. 3, pp. 20-32, September 2012.

[21] J. Yao, Z. Jiao, and D. Ma, "Extended-state-observer-based output feedback nonlinear robust control of hydraulic systems with backstepping," Industrial Electronics, IEEE Transactions on, vol. 61, no. 11, pp. 62856293, 2014.

[22] J. Yao, Z. Jiao, D. Ma, and L. Yan, "High-accuracy tracking control of hydraulic rotary actuators with modeling uncertainties," Mechatronics, IEEE/ASME Transactions on, vol. 19, no. 2, pp. 633-641, 2014.

[23] J. B. Kuipers, Quaternions and Rotation Sequences, P. U. Press, Ed., 1998.

[24] J. Diebel, "Representing Attitude: Euler Angles, Unit Quaternions, and Rotation Vectors," 2006.

[25] H. Schaub and J. L. Junkins, "Stereographic Orientation Parameters for Attitude Dynamics: A generalization of the Rodrigues Parameters," Journal of the Astronautical Sciences, vol. 44, pp. 1-20, 1996.

[26] G. J. Bierman, Factorization Methods for Discrete Sequential Estimation. Academic Press, 1977

[27] C. L. Thornton, "Triangular Covariance Factorizations for Kalman Filtering," Ph.D. dissertation, California Institute of Technology, 1976.

[28] A. W. Bojanczyk, R. P. Brent, P. van Dooren and F. H. de Hoog, "A note on downdating the Cholesky factorization," SIAM J. Sci. Stat. Comput, vol. 8, pp. 210-221, 1987.

[29] A. Ukil, V. H. Shah, and B. Deck, "Fast computation of arctangent functions for embedded applications: A comparative analysis," 2011.

[30] T. Bresciani, "Modelling, identification and control of a quadrotor helicopter," Ph.D. dissertation, Lund University, 2008.
[31] E. Fresk and G. Nikolakopoulos, "Experimental Model Derivation and Control of a Variable Pitch Propeller Quadrotor," IEEE Multi-Conference on Systems and Control, 2014.

[32] R. Van Der Merwe, E. Wan et al., "The square-root unscented Kalman filter for state and parameter-estimation," in Acoustics, Speech, and Signal Processing, 2001. Proceedings.(ICASSP'01). 2001 IEEE International Conference on, vol. 6. IEEE, 2001, pp. 3461-3464.

[33] GitHub: Comparison of UKF CKF EKF, http://www.github.com/CEGLTU/Comparison-of-UKF-CKF-EKF.

[34] ChibiOS/RT, http://chibios.org/dokuwiki/doku.php.

[35] S. O. Madgwick, "An efficient orientation filter for inertial and inertial/magnetic sensor arrays," Tech. Rep., 2010.

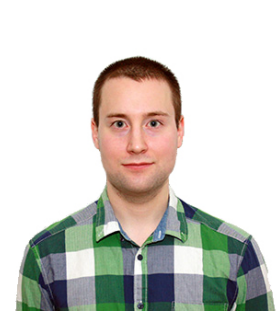

Emil Fresk Emil Fresk is a $\mathrm{PhD}$ candidate at the Control Engineering Group at the Department of Computer Science, Electrical and Space Engineering, Luleå, Sweden. His main focus of interest is in Cooperative Unmanned Aerial Vehicles and estimation of localization based on image, sonar and IMUs. He is also a principal technical coordinator for LTU in the Horizon 2020 project AEROWORKS, with a specific focus in creating generalized and adaptable estimation and control schemes for UAVs.

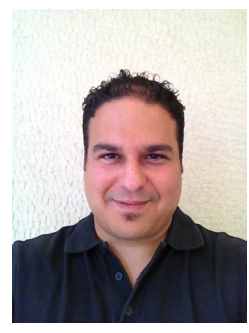

George Nikolakopoulos Associate Professor at the Department of Computer Science, Electrical and Space Engineering at Luleå University of Technology, Luleå, Sweden. His work is focusing in the area of Robotics, Control Applications. George is the coordinator of H2020-ICT AEROWORKS project in the field of Aerial Collaborative UAVs and H2020SPIRE project DISIRE in the field of Integrated Process Control. In 2014, he has been nominated as LTUs Wallenberg candidate, one out of three nominations from the university and 16 in total engineering nominations in Sweden. George has received the Information Societies Technologies (IST) Prize Award for the best paper that Promotes the scopes of the European IST (ICT nowadays). His publications in the field of UAVs have received top recognition from the related scientific community and include more than 150 published International Journals and Conferences in the fields of his interest.

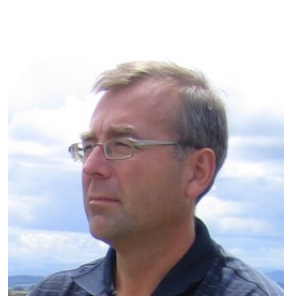

Thomas Gustafsson Thomas Gustafsson is Chaired Professor of Automatic Control in the Department of Computer Science, Electrical and Space Engineering, Luleå University of Technology (LTU), Sweden. His current research interests include estimation and control applied in process industries and field robotics. He has held visiting positions at Monash University and University of Newcastle, both in Australia. He received an M.Sc. in Mechanical Engineering (1982) and Ph.D. in Automatic Control (1993) both from LTU. He is a board member of Centre for Distance Spanning Technology and member of the executive committee for Process IT Innovations and Hjalmar Lundbohm Research Centre. 